\title{
Evolución glaciar y morfodinámica periglaciar en la vertiente asturiana del Puerto de Ventana (Montañas Cantábricas)
}

\section{Glacial evolution and periglacial morphodynamics in the Asturian area of Puerto de Ventana (Cantabrian Mountains)}

\author{
Benjamín GONZÁLEZ-DÍAZ* , Jesús RUIZ-FERNÁNDEZ ${ }^{1}$, Cristina GARCÍA- \\ HERNÁNDEZ ${ }^{1}$, Rosana MENÉNDEZ-DUARTE ${ }^{2}$, José Antonio GONZÁLEZ-DÍAZ ${ }^{1}$ \\ ${ }^{1}$ Departamento de Geografía y Ordenación del territorio, Universidad de Oviedo, Oviedo, España. \\ ${ }^{2}$ Departamento de Geología, Universidad de Oviedo, Oviedo, España. \\ *Autor de contacto: gonzalezbenjamin@uniovi.es
}

https://doi.org/10.17979/cadlaxe.2021.43.0.8841 recibido: 15/12/2021 aceptado: 31/12/2021

\begin{abstract}
Despite the recent advances in glacial and periglacial studies in the Cantabrian Mountains, the focus of research has been mainly on the highest altitude areas, while areas such as the centralwestern sector of the Asturian mountains have received less attention. In this sector, Puerto de Ventana constitutes a paradigmatic area due to its altimetric and lithostratigraphic characteristics. In this study, both glacial and periglacial morphologies of the Puerto de Ventana have been identified and mapped, subsequently turning the information into a GIS and proceeding to the paleoglacial reconstruction and to the calculation of paleoELAs. The results show the existence of three stages in the glacial evolution of the area: Maximum Ice Extension Stage (MEH), Internal Stage and Cirque Stage. Through these phases the altitude of the paleoELAs goes from 1400-1557 in the MEH to 1515-1649 and 1650-1838 $\mathrm{m}$ in the following stages (depending on the method used). Likewise, from an initial glacial surface of 491.3 ha and an ice volume of $510.7 \mathrm{hm}^{3}$ in the $\mathrm{MEH}$, the second and third stages show a reduction of $90.25 \%$ and $96.28 \%$ in volume, respectively. Moreover, the study of periglacial morphodynamics allowed to identify both relict morphologies whose genesis is highly conditioned by the existing lithology in the area, and functional morphologies showing a weak geomorphological activity and a marked seasonal character compared to other areas of the Cantabrian Mountains.
\end{abstract}

Keywords: GIS, paleoglaciers, paleoELAs, periglacial processes and landforms, Ventana Pass 


\section{Resumen}

A pesar del avance reciente de los estudios sobre glaciarismo y periglaciarismo en las Montañas Cantábricas, el foco de las investigaciones se ha puesto principalmente en los conjuntos de mayor altitud, mientras áreas como el sector centro-occidental de la montaña asturiana han recibido menor atención. En este ámbito, el Puerto de Ventana constituye un área paradigmática debido a sus características altimétricas y litoestratigráficas. En este estudio se han identificado y cartografiado las formas de origen glaciar y periglaciar de la vertiente asturiana de dicho puerto, volcando posteriormente la información en un SIG y procediendo a la reconstrucción paleoglaciar y al cálculo de paleoELAs. Los resultados arrojan la existencia de tres fases en la evolución glaciar del área: Fase de Máxima extensión de los Hielos (MEH), Fase Interna y Fase de Circos. A través de estas fases, la altitud de las paleoELAs va pasando en función del método de los 1.400-1.557 en la MEH a los 1.515-1.649 y 1.650-1.838 m en las siguientes. Igualmente, a partir de una superficie glaciada inicial de 491,3 ha y un volumen de hielo de $510,7 \mathrm{hm}^{3}$, la segunda y tercera fases muestran una reducción del 90,25 \% y el 96,28 \% del volumen, respectivamente. El estudio de la morfodinámica periglaciar, por su parte, ha permitido identificar morfologías relictas cuya génesis se encuentra altamente condicionada por la litología existente en el área, y geoformas funcionales de dinámica atenuada y marcado carácter estacional, en comparación con otras áreas de las Montañas Cantábricas.

Palabras clave: SIG, paleoglaciares, paleoELAs, formas y procesos periglaciares, Puerto de Ventana 


\section{INTRODUCCIÓN}

Los estudios de geomorfología glaciar y periglaciar han experimentado un importante avance en España a partir de la segunda mitad del S. XX, como atestiguan los ejemplos representados por ciertas obras de síntesis, como las coordinadas por GÓMEZ-ORTIZ et al. (1994), GÓMEZ-ORTIZ y PÉREZ-ALBERTI (1998) y OLIVA et al. (2016, 2022). Este avance, se ha producido de forma paralela al desarrollo de los diferentes departamentos universitarios relacionados con el estudio de dichas disciplinas, lo que a su vez permitió el surgimiento y consolidación de diversos grupos de trabajo vinculados territorialmente con los principales conjuntos montañosos afectados por procesos fríos. Ello dio lugar a numerosas publicaciones sobre glaciarismo y periglaciarismo que, si bien conjuntamente tienen una amplia representatividad espacial, se centran preferentemente en los ámbitos de mayor interés de los grandes conjuntos montañosos ibéricos. Por tanto, aún quedan numerosos sectores por estudiar desde un punto de vista específico, algunos de los cuales plantean, además, interrogantes significativos en el marco del glaciarismo y el periglaciarismo ibérico, debido a su baja cota altimétrica y carácter marginal, o bien por la presencia de procesos activos.

En este sentido, en las Montañas Cantábricas, el foco de estas investigaciones se ha puesto preferentemente en ámbitos de alta montaña como los Picos de Europa, así como determinados sectores de las montañas leonesas, cántabras, burgalesas y palentinas (ej. FROCHOSO, 1980, 1990; CASTAÑÓN, 1989; RODRÍGUEZ PÉREZ, 1995; CASTAÑÓN y FROCHOSO, 1994, 1998; JIMÉNEZ, 1996; GALE y HOARE, 1997; FROCHOSO y CASTAÑÓN, 1998; GARCÍA DE CELIS y MARTÍNEZ FERNÁNDEZ, 2002; GONZÁLEZ-GUTIÉRREZ, 2002; JIMÉNEZ y FARIAS, 2002; SERRANO y GONZÁLEZ-TRUEBA, 2004A; GONZÁLEZ-TRUEBA, 2005, 2007; PELLITERO, 2009; SANTOS-GONZÁLEZ, 2010; SANTOS-GONZÁLEZ et al., 2009, 2013; MORENO et al., 2010; GÓMEZ-VILLAR et al., 2011; 2015; PELLITERO et al., 2011, 2013; SERRANO et al., 2012, 2013; JIMÉNEZ et al., 2013; RODRÍGUEZ-RODRÍGUEZ et al., 2015; PISABARRO et al., 2017; RUIZFERNÁNDEZ et al. 2016; 2017). En cambio, otras áreas han recibido menor atención científica, como por ejemplo el sector centro-occidental de Asturias (RUIZFERNÁNDEZ et al., 2022), cuyo despegue en lo que se refiere a los estudios de glaciarismo cuaternario (exceptuando algunas obras pioneras), se produjo en las dos últimas décadas del S. XX.

A partir de este momento, se sucedieron diversas investigaciones centradas en el estudio del Sinclinal de Saliencia, el Macizo de las Ubiñas, el Macizo de Somiedo, el sector de Valgrande-Pajares, el Valle de Degaña, el Valle del Pigüeña, el Alto Valle del Narcea y el Puerto de Leitariegos (MUÑOZ-JIMÉNEZ, 1980, CASTAÑÓN, 1983, 1989; ALONSO y FLOR, 1987; ALONSO, 1989, 1991, 1994; FELICÍSIMO y ALONSO, 1988; MENÉNDEZ DUARTE, 1995; MENÉNDEZ DUARTE y MARQUÍNEZ, 1996; GARCÍA DE CELIS, 1997; RUIZ-FERNÁNDEZ et al., 2009; GALLINAR et al., 2014). Dichos estudios, se centraron en la descripción de las huellas glaciares 
detectadas, la extensión de los glaciares durante la fase de máximo avance dentro de la última glaciación, así como la aportación de modelos evolutivos a partir de cronologías relativas. Pese a ello, el conocimiento de la morfología y evolución glaciar del sector centro-occidental de Asturias aún es escaso: muchos valles y cordales montañosos continúan sin ser estudiados en detalle. Además, no se han aportado hasta la fecha paleoELAs ni datos geocronológicos absolutos sobre este ámbito. Las paleoELAs más cercanas son las calculadas en el estudio de SANTOS-GONZÁLEZ et al. (2013), circunscrito a sectores próximos de la montaña leonesa. Por su parte, las aproximaciones cronológicas efectuadas en los estudios citados anteriormente se han basado esencialmente en criterios morfoestatigráficos y en correlaciones con lo acontecido en otros conjuntos montañosos peninsulares. Ha de tenerse en cuenta, no obstante, que casi la totalidad de estas publicaciones son anteriores a la generalización de las técnicas de datación absoluta y de los modelos de reconstrucción paleoglaciar a través de técnicas SIG.

En cuanto al estudio de la dinámica periglaciar en las Montañas Cantábricas, si bien hay algún precedente incluso en la segunda mitad del S. XIX (se trata generalmente de alusiones puntuales e inespecíficas a formas y depósitos periglaciares; ver al respecto el trabajo de síntesis de CASTAÑÓN y FROCHOSO, 1994), su despegue significativo se produjo en las tres últimas décadas del S. XX. En un primer momento, una gran parte de estas investigaciones se centró en el estudio de formas periglaciares relictas, heredadas de los periodos fríos finipleistocenos (ej. ALONSO, 1989; GARCÍA DE CELIS, 1991, 1997, 2002; CASTAÑÓN y FROCHOSO, 1994; RODRÍGUEZ-PÉREZ, 1995, 1998, 2015; JIMÉNEZ, 1996; GONZÁLEZ-GUTIÉRREZ, 2002; REDONDO-VEGA et al., 2004; GÓMEZ-VILLAR et al., 2011; SANTOS-GONZÁLEZ, 2010; PELLITERO et al., 2011; RUIZ-FERNÁNDEZ, 2015; RUIZ-FERNÁNDEZ et al., 2016). Con la excepción de algunos trabajos precursores como el de BROSCHE (1978), es a partir de los años finales de la pasada centuria cuando se van sucediendo importantes avances en el conocimiento de la morfodinámica periglaciar activa que se desarrolla en los sectores de mayor altitud de las Montañas Cantábricas, como por ejemplo los Picos de Europa (ej. CASTAÑÓN y FROCHOSO, 1998; SERRANO y GONZÁLEZ-TRUEBA, 2004A, GONZÁLEZ-TRUEBA， 2007; GONZÁLEZ-TRUEBA y SERRANO， 2010; PELLITERO, 2012; GALLINAR et al., 2014; RUIZ-FERNÁNDEZ et al., 2014, 2017; RUIZ-FERNÁNDEZ, 2015). Por último, otro de los enfoques desarrollado en estas investigaciones es el del estudio de las condiciones morfoclimáticas que posibilitan dicha morfodinámica, específicamente el régimen térmico del suelo y también la evolución anual del manto nival en las áreas de alta montaña (ej. CASTAÑÓN y FROCHOSO, 1998; SANTOS-GONZÁLEZ et al., 2009; GONZÁLEZ-TRUEBA y SERRANO, 2010; PELLITERO, 2012; RUIZ-FERNÁNDEZ et al., 2014, 2017; PISABARRO et al., 2017). Sin embargo, pese a contar un número importante de trabajos de calidad, el estudio del periglaciarismo en las Montañas Cantábricas aún cuenta con extensas áreas carentes de estudios específicos. Este es el caso, nuevamente, de las montañas centro-occidentales de Asturias, donde cabe citar algunos estudios (ALONSO, 1989; GARCÍA DE CELIS, 1997; RODRÍGUEZ PÉREZ, 1998; 2012, 
2015; ALONSO y TROMBOTTO, 2009; GALLINAR et al., 2014). También es necesario resaltar que, en el caso de las formas periglaciares relictas, la casi inexistencia de cronologías absolutas en las Montañas Cantábricas constituye otra laguna importante que deberá ser paliada de forma progresiva en los próximos años (OLIVA et al., 2016).

Teniendo en cuenta lo comentado en los párrafos anteriores, en esta aportación se pretende estudiar la morfología y la evolución glaciar, así como la morfodinámica periglaciar del Puerto de Ventana. Esta área constituye un sector paradigmático dentro de las montañas centro-occidentales asturianas, debido a sus características litoestratigráficas (roquedo principalmente silíceo) y altimétricas (altitudes máximas moderadas). A partir de dicho objetivo general, este estudio pretende dar respuesta a los siguientes objetivos específicos:

i) Cartografiar las formas de relieve de origen glaciar y periglaciar presentes en el área de estudio.

ii) Identificar las fases glaciares reconocibles a partir de criterios morfoestatigráficos, así como reconstruir los paleoglaciares existentes en cada una, calculando las respectivas paleoELAS a partir de los métodos AABR (Area Altitude Balance Ratio), AAR (Acumulation Area Ratio) y THAR (Terminus Headwall Altitude Ratio), junto a los correspondientes espesores y volúmenes del hielo.

iii) Estudiar las formas $\mathrm{y}$ procesos periglaciares existentes, tanto activos/semiactivos como relictos, así como sus implicaciones ambientales.

\section{2. ÁREA DE ESTUDIO}

El Puerto de Ventana se encuentra en el límite administrativo de las comunidades autónomas de Asturias y Castilla y León. Concretamente, el sector analizado se corresponde con la vertiente asturiana de dicho puerto (coordenadas 4303'29' y 434'03' de latitud N, 60'30' y 6 $6^{\circ}$ '07' de longitud W; Figura 1). Atendiendo a la orografía, el área de estudio está compuesta por cuatro cimas principales, que de Este a Oeste son: el Boquerón de Ventana (1.713 m), el Pico del Cuervo (1.826 m), el Pico de Ferreirúa $(1.977 \mathrm{~m})$ y el Pico del Águila $(1.822 \mathrm{~m})$. En cuanto a la hidrografía, el río la Puerca da lugar al eje principal del valle estudiado. Este río, a su vez, es tributario del río Páramo, que nace en un valle paralelo situado inmediatamente al Este del anterior. También cabe mencionar el arroyo de la Ortigosa, que confluye con el río la Puerca por el Oeste, naciendo en las inmediaciones del Collado de Ferreirúa (1.629 m).

Desde el punto de vista litológico, la cabecera glaciar está labrada sobre las cuarcitas de la Fm. Barrios (Ordovícico), mientras que en el área del Collado de Ferreirúa afloran las pizarras negras de la Fm. Formigoso (Silúrico Inferior). Por su parte, los sectores intermedios de la misma vertiente están integrados por las areniscas y pizarras de la Fm. Oville (Ordovícico Inferior), y por las calizas de la Fm. Láncara (Cámbrico Inferior). En el sector inferior del valle afloran las lutitas de la Fm. Santo Emiliano (Carbonífero 
Superior). Todos estos materiales forman una escama cabalgante que a su vez está cabalgada por el norte por otra integrada en el área frontal por una unidad de conglomerados, areniscas, pizarras y capas de carbón (Carbonífero Superior) (TRUYOLS et al., 1982). Geomorfológicamente, dicho sector presenta cuantiosas formas de origen glaciar, tanto erosivas (circos glaciares, cubetas de sobreexcavación, umbrales, rocas aborregadas) como de tipo sedimentario (depósitos en forma de arcos y cordones morrénicos). También abundan formas y depósitos periglaciares y nivales activos o semiactivos (canales de aludes, sectores proximales de conos y taludes de derrubios, y solifluxiones), así como relictos (campos de bloques y un glaciar rocoso).
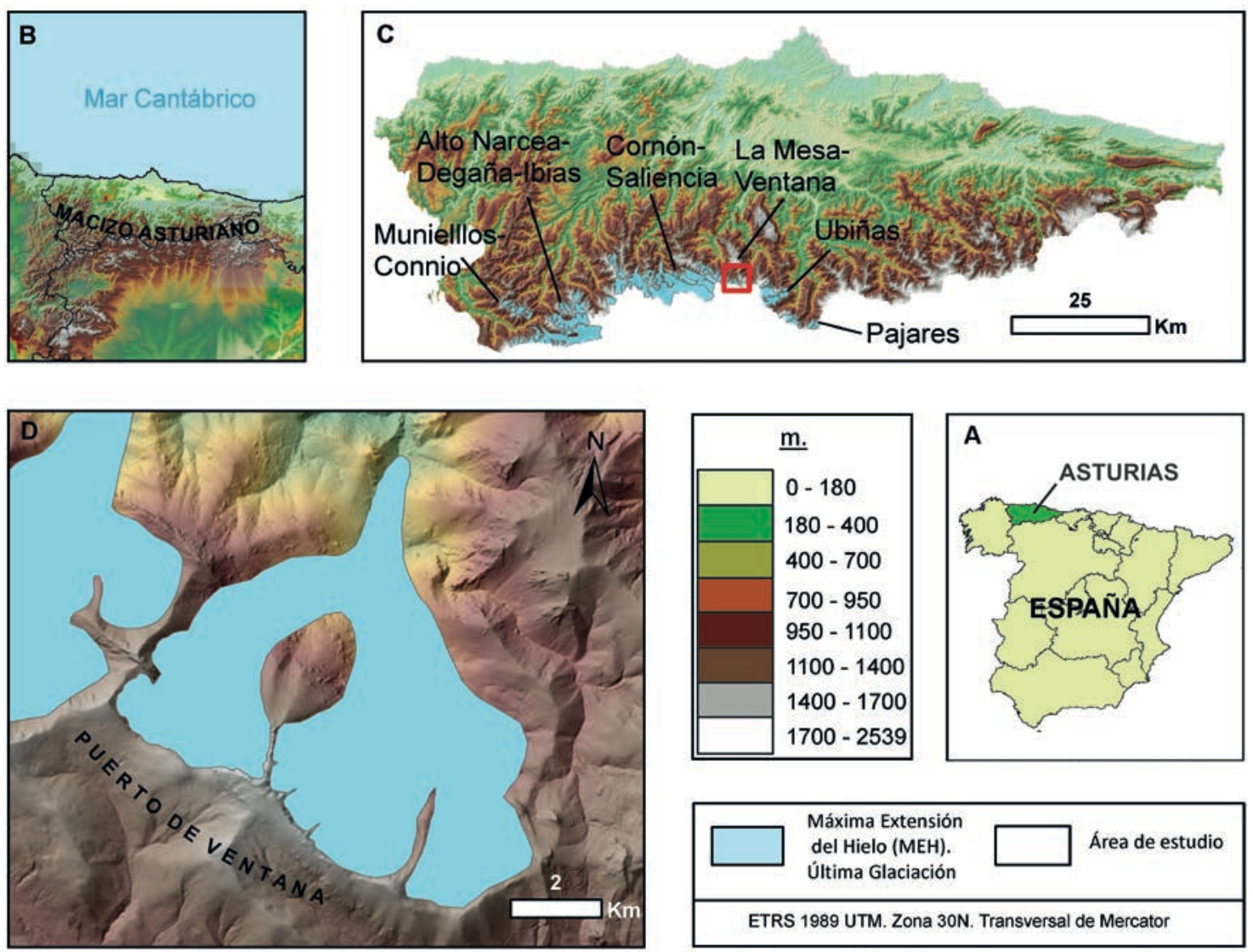

Figura 1. Localización de la vertiente asturiana del Puerto de Ventana. Se representa también la extensión máxima de los glaciares del sector centro-occidental de Asturias durante la Última Glaciación (C).

En cuanto al clima, dentro de la clasificación de Köppen el área de estudio se encuadra en los tipos Cfsc y Dfsc. El primero es un clima fresco característico de las áreas montañosas situadas entre 1.000 y 1.500 m de altitud (MUÑOZ JIMÉNEZ, 1982). Por su parte, el tipo Dfsc es propio de los sectores de mayor altitud del Macizo Asturiano (por encima de $1.500 \mathrm{~m}$ ), definidos por condiciones muy húmedas y frías en las que la temperatura media puede ser inferior a $0^{\circ} \mathrm{C}$ durante varios meses al año (MUÑOZ JIMÉNEZ, 1982). Desde el punto de vista biogeográfico, el área de estudio forma parte de la región Eurosiberiana, y de la subprovincia Cantabroatlántica, teniendo presentes en el área de estudio tres pisos biogeográficos. El piso basal o colino, entre el nivel del 
mar y los $\sim 700$ metros de altitud, se encuentra dominado por los bosques mixtos, los castañedos (Castanea sativa) y los robledales de Quercus robur. Por su parte, el piso montano está dominado por robledales albares (Quercus petraea) y hayedos (Fagus sylvática). Como en el piso basal, las formaciones forestales del piso montano cedieron paso al establecimiento de áreas de pasto para el desarrollo de la actividad ganadera, que hoy está en fuerte retroceso y ha dado lugar a la proliferación de amplias superficies de matorral. Por encima de ellos, los abedulares orocantábricos (Betula celtibérica) se sitúan en el límite superior del bosque dentro de esta área, que varía entre 1.500 y 1.700 $\mathrm{m}$ en función de la localización concreta. Por tanto, debido a las actividades agroganaderas tradicionales, dicho límite se encuentra rebajado en algunos sectores con respecto a su altitud potencial. Los sectores culminantes del Puerto de Ventana quedarían integrados dentro del piso subalpino, caracterizado por la ausencia de vegetación arbórea y por la adaptación del resto de formaciones a un ambiente con fuertes vientos e importante innivación.

Por otra parte, cabe señalar la inexistencia de núcleos de población dentro del ámbito de estudio. El núcleo más próximo en la vertiente asturiana es Páramo, situado a $10 \mathrm{~km}$ al norte. A $8,2 \mathrm{~km}$ al Sur, ya en la vertiente leonesa, está Torrestío. Además de la ganadería, ya mencionada, las principales actividades humanas de este entorno son la minería de carbón que existió en las inmediaciones (ej: mina explotada hasta finales del S. XX en el sector de Busbudín, al noreste del Puerto de Ventana), el senderismo y los deportes de montaña, así como su importante función como vía de paso preferente en este sector de la divisoria hidrográfica, a través de la carretera AS-228. De hecho, hasta finales del S. XVIII, el Puerto de Ventana constituyó el principal paso histórico de Asturias a la Meseta (GALLINAR et al., 2019). Desde el año 2006, el sector asturiano del Puerto de Ventana forma parte del Parque Natural de Las Ubiñas - La Mesa, mientras que la vertiente leonesa está integrada en el Parque Natural de Babia y Luna (declarado en el año 2015). A su vez, el área de estudio está incluida dentro de la Reserva de la Biosfera de Las Ubiñas - La Mesa (año 2012).

\section{METODOLOGÍA}

La metodología de este estudio se basa en la realización de transectos sistemáticos a lo largo del área seleccionada, con el fin de identificar las huellas glaciares cuaternarias que se conservan, así como los procesos y formas periglaciares y nivales existentes. Dicha información, se sintetiza en las Tablas 1 y 2 . El cálculo de datos básicos de superficie, longitud y anchura de las geoformas analizadas se ha obtenido a partir del software ArcGis de ESRI en su versión 10.5. Por otra parte, las diferencias entre las formas y procesos periglaciares relictos y actuales presentes entre las vertientes leonesa y asturiana, se ha sintetizado en un perfil geomorfológico (Figura 10). 


\subsection{Cartografía geomorfológica}

La información geomorfológica recopilada se ha volcado también en un SIG, a partir del cual se ha confeccionado la cartografía geomorfológica asociada. Para la elaboración de la base cartográfica se utilizaron como fuentes el Mapa Geológico Nacional a escala 1:50.000 (Serie MAGNA, hoja $n^{\circ} 77$ de La Plaza; TRUYOLS et al, 1987), así como el mismo sector del Mapa Topográfico Nacional a escala 1:25.000 en formato ráster, y las ortofotografías del Plan Nacional de Ortofotografía Aérea (PNOA) de máxima actualidad, así como el Modelo Digital del Terreno de $5 \mathrm{~m}$ de resolución (MDT05). La salida gráfica final del mapa se realizó mediante el software Adobe Ilustrator CS6 (Figura 2). El método cartográfico utilizado es el RCP 77 del Centre National de la Recherche Scientifique (CNRS; JOLY, 1997) si bien se han incorporado adaptaciones propias (RUIZ-FERNÁNDEZ, 2011).

\subsection{Reconstrucción paleoglaciar y cálculo de paleoELAs}

Para efectuar la reconstrucción de los paleoglaciares identificados se empleó la herramienta Glare (PELLITERO et al., 2016) que, a través de la interpolación de las formas glaciares digitalizadas con el relieve representado en un MDT, nos permitió la reconstrucción de paleo-topografías, así como la creación de modelos espaciales de espesores y la estimación de volúmenes. Por último, se procedió al cálculo de paleoELAS mediante el método AABR (Area Altitude Balance Ratio), y complementariamente con los métodos AAR (Acumulation Area Ratio) y THAR (Terminus Headwall Altitude Ratio). El primero de ambos métodos (AABR o simplemente BR) tiene en cuenta la hipsometría glaciar (la distribución de la superficie en relación con el rango altitudinal) y el balance de masa (BENN y LEHMKUHL, 2000; VIEIRA, 2008). Fue definido por Osmaston en 1965 precisamente para solucionar los problemas generados en el cálculo de paleoELAs de glaciares con curvas hipsométricas asimétricas (OSMASTON, 2005; VIEIRA, 2008), y posteriormente desarrollado por FURBISH y ANDREWS (1984). BENN y GEMMELL (1997) y OSMASTON (2005) proporcionan hojas de cálculo que permiten obtener ELAs con este método, mientras que REA (2009) ha calculado la ELA de glaciares activos de diferentes regiones (Noruega, Ártico Canadiense, Los Alpes, Asia Central, Islas Svalbard, etc.), lo que reviste gran interés para su comparación con glaciares desaparecidos. En nuestro caso, de acuerdo con REA (2009), se ha utilizado un AABR de 1,7. Por su parte, el método AAR fue propuesto por Ritcher y Brückner y utilizado esencialmente en glaciares estables de latitudes medias y altas, que tienen por tanto rangos para el área de acumulación con respecto a la superficie total (acumulation area ratio o AAR) de 0,5 a 0,8 (MEIERDING, 1982; CARRIVICK y BREWER, 2004; SERRANO y GONZÁLEZ-TRUEBA, 2004B; VIEIRA, 2008; COWTON et al., 2009; REA, 2009). Por debajo de 0,5 los glaciares tendrían balance de masa negativo y por encima de 0,8 positivo; siendo los valores AAR más habituales para glaciares alpinos de latitudes medias los comprendidos entre 0,55 y 0,65 (ALLEN, 1998; SERRANO y GONZÁLEZ-TRUEBA, 2004A; BENN et al., 2005; GONZÁLEZ-TRUEBA, 2007). 
Es decir, en situaciones estacionarias el área de acumulación de estos glaciares representa entre el 55 y el $65 \%$ de su superficie total (SERRANO y GONZÁLEZTRUEBA, 2004B; VIEIRA, 2008). Según diversos autores (BENN y EVANS, 1998; REA, 2009), el mayor inconveniente de este método es que no tiene en cuenta la hipsometría glaciar, que puede tener una influencia muy importante en la ELA. Por tanto, su aplicación a glaciares complejos como campos de hielo (ej: Picos de Europa, Sanabria, Serra da Estrela) y glaciares de piedemonte, es especialmente problemática debido a las peculiares características hipsométricas de estos aparatos (VIEIRA, 2008). Por este motivo, prácticamente ha sido sustituido por el AABR (REA, 2009). Por su parte, el método THAR asume que la paleoELA se encuentra a una proporción dada de la distancia vertical que separa el punto más bajo y el más alto de un glaciar. Para ello se aplican proporciones diversas, aunque las más comunes son de 0,6 a 0,4 (BENN et al., 2005). Por tanto, no tiene en cuenta las variaciones topográficas que generalmente hay en los valles glaciares, de gran influencia en el balance de masa y en la ELA. A su favor tiene que es un método de fácil aplicación, ya que se basa en una operación sencilla y fácil de aplicar. Los mejores resultados se obtienen en pequeños glaciares de geometría regular (PORTER, 1975; MEIERDING, 1982; CARRIVICK y BREWER, 2004). En nuestro caso, se ha aplicado una ratio de 0,4 .

\section{RESULTADOS Y DISCUSIÓN}

\subsection{La morfología y la evolución glaciar}

Dentro del área de estudio de este trabajo se han identificado formas de origen glaciar de tipo erosivo (circos glaciares, umbrales, rocas aborregadas, estrías, y una cubeta de sobreexcavación glaciar), junto a depósitos glaciares en forma de arcos y cordones morrénicos, organizados en tres generaciones distintas (Figura 2). En los siguientes subapartados se desarrollarán las características de cada uno de ellos.

\subsubsection{Formas erosivas}

La cabecera glaciar del área de estudio está compuesta por un total de ocho circos glaciares labrados principalmente sobre litología cuarcítica, cuyas altitudes máximas están comprendidas entre 1.713 y $1.977 \mathrm{~m}$, mientras que la anchura oscila entre $200 \mathrm{~m}$ y $800 \mathrm{~m}$, la longitud entre 178 y $469 \mathrm{~m}$, y el desnivel (o diferencia entre el punto más alto del cordal culminante y el más bajo del interior del circo) varía entre 123 y $320 \mathrm{~m}$ (Figura 3A). Dichos parámetros, junto con las orientaciones dominantes de los circos del área de estudio ( $\mathrm{N}, \mathrm{NW}$ y NE), concuerdan con los rangos identificados en un estudio morfométrico de circos glaciares desarrollado por RUIZ-FERNÁNDEZ et al. (2009) en varias sierras del suroccidente de Asturias.

Son comunes, en determinados sectores del área de estudio, como el de Refuexu, los umbrales labrados por el paso del hielo sobre capas cuarcíticas (Figura 3B). También se han encontrado rocas aborregadas con el perfil romo y el pulido característico de origen glaciar. 


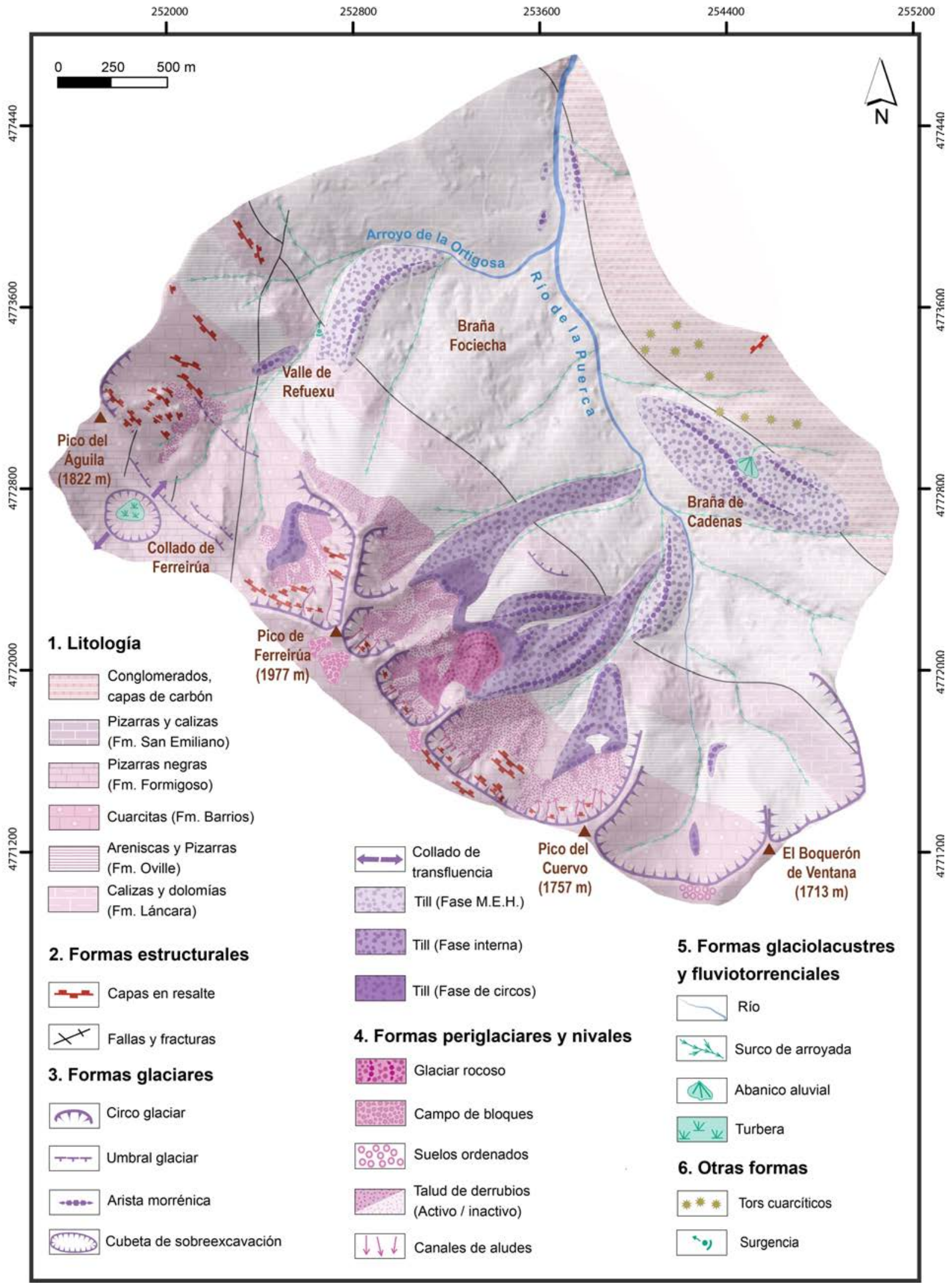

Figura 2. Mapa geomorfológico del área de estudio. 
Tanto en los umbrales como en las rocas aborregadas es posible distinguir estrías glaciares que confirman el paso del hielo. Al norte del Collado de Ferreirúa (1.722 m), se generó una cubeta de sobreexcavación glaciar cerrada por un umbral y posteriormente colmatada por una turbera. Este collado, a su vez, constituyó un área de transfluencia glaciar con respecto a la vertiente leonesa (Figura 3B). En su tramo inferior, el valle glaciar de Ventana tiene una morfología en $U$ incipiente, aunque sin dar lugar a una artesa bien definida, debido a la deleznabilidad de las capas rocosas que conforman los sectores situados a ambos flancos del eje del valle.
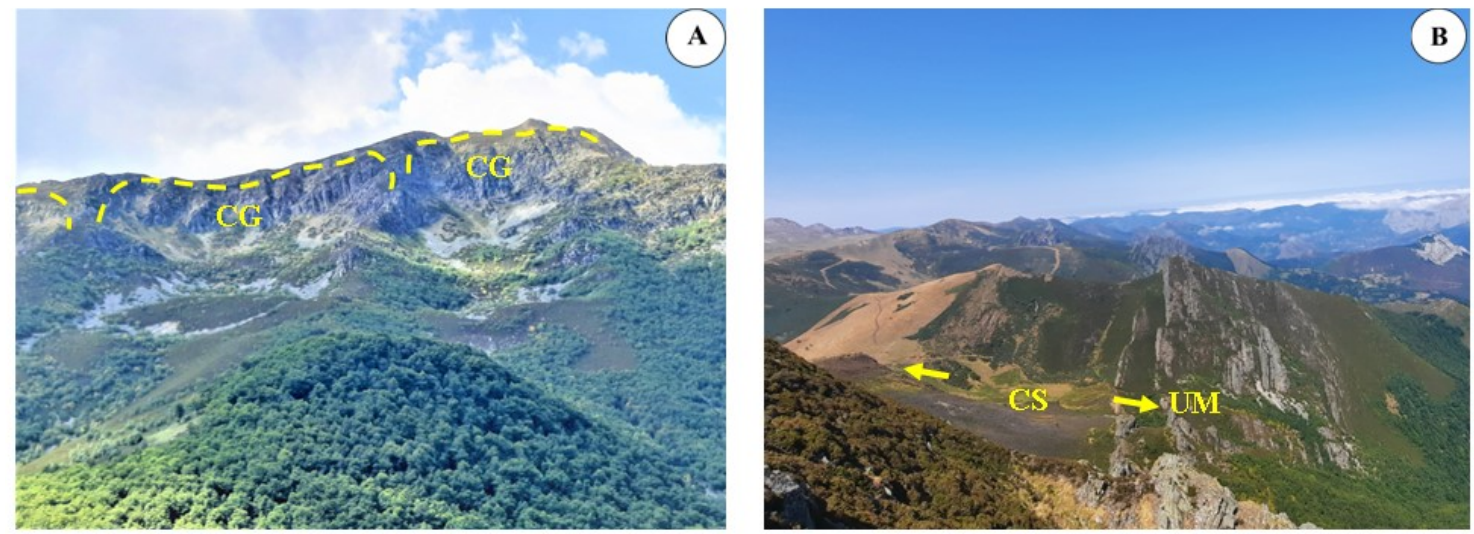

Figura 3. Formas erosivas de origen glaciar: A) Circos glaciares (CG) al este del Pico Ferreirúa, B) Cubeta de sobreexcavación glaciar (CS) que se corresponde con un collado de transfluencia señalado por las flechas amarillas, y umbrales (UM) situados en el área del Collado de Ferreirúa.

\subsubsection{Depósitos glaciares}

En cuanto a los depósitos de origen glaciar, en el Puerto de Ventana se conservan tres generaciones sucesivas de complejos morrénicos (Figura 2): 1) complejos morrénicos externos, 2) complejos morrénicos internos y, 3) complejos morrénicos de pie de circo. A continuación, se describirá cada uno de ellos.

4.1.2.1. Complejos morrénicos externos. Estos complejos morrénicos están integrados por siete morrenas que el glaciar depositó durante la fase de máxima extensión del hielo $(\mathrm{MEH})$ dentro de la Última Glaciación. De ellas, tres retazos de modestas dimensiones (al haber sido parcialmente desmantelados), se sitúan en posiciones más externas con respecto al Valle de la Puerca y en ambas márgenes del mismo, permitiendo reconstruir el frente del paleoglaciar de Ventana (Figura 2). Adosada a la margen derecha de dicho curso fluvial, se encuentra la morrena lateral más extensa de estos complejos y la mejor conservada de las tres mencionadas, que se desarrolla entre los $1.030 \mathrm{~m}$ y $1.100 \mathrm{~m}$ de altitud (Figura 4A). En su tramo final, dicha morrena queda individualizada de la vertiente, evidenciando un espesor visible de entre $15 \mathrm{~m}$ y $16 \mathrm{~m}$. Sobre su superficie se conservan bloques de cuarcita de tamaño métrico (Figura 4B), hecho que contrasta claramente con la litología pizarrosa de este tramo del valle, siendo la extensión estimada que ocupa el till de 2 ha. En la margen opuesta del río, se encuentran los otros 
dos retazos mencionados, de dimensiones muy reducidas, en los que se han encontrado pequeños bloques y cantos de litología calcárea y cuarcítica, que permiten confirmar el origen glaciar de los mismos. Aguas abajo se aprecia un cambio morfológico importante en el valle, que pasa a tener un marcado perfil en $\mathrm{V}$, lo que evidencia que el paleoglaciar no descendió aguas abajo del complejo morrénico descrito.
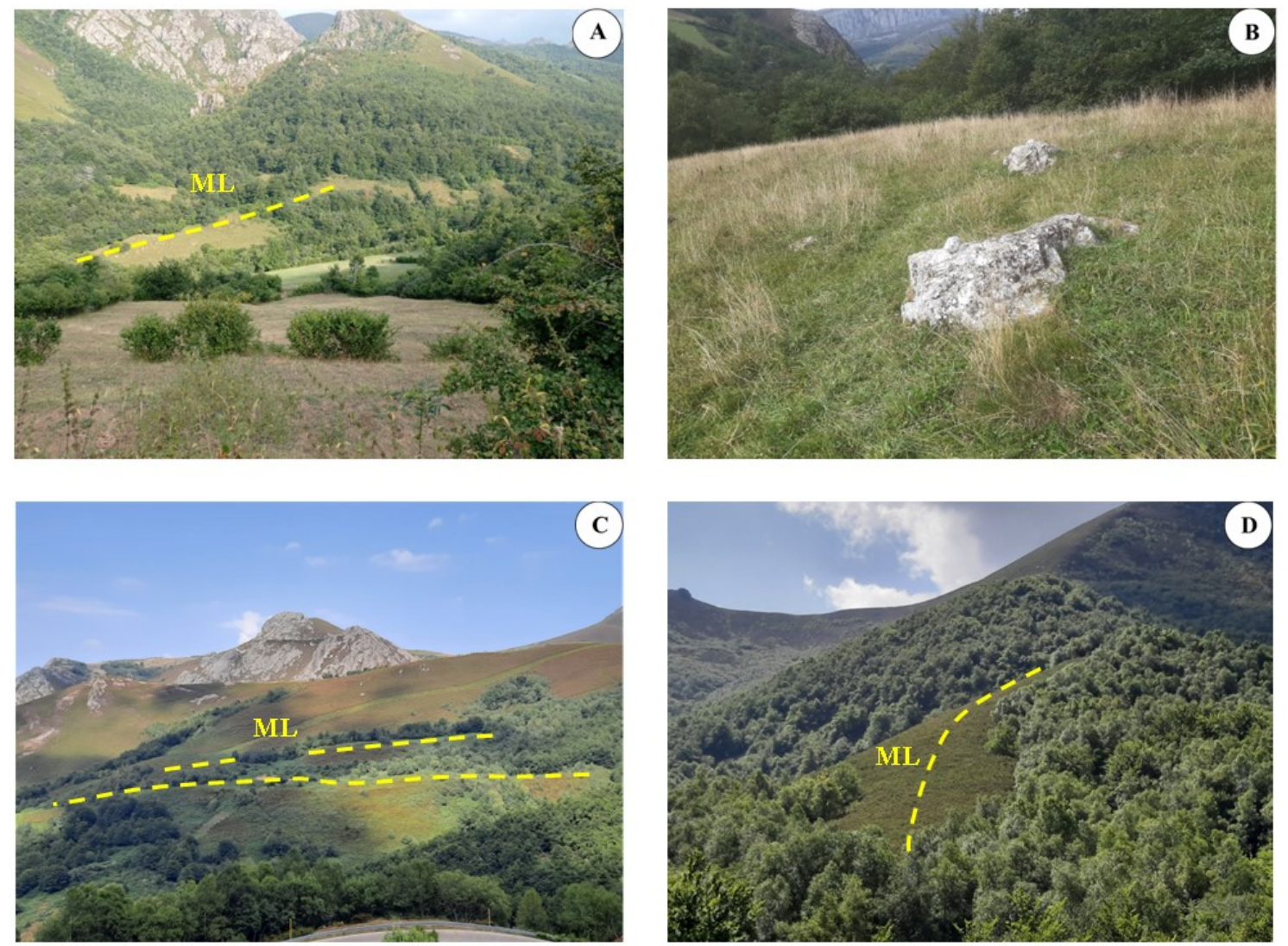

Figura 4. Morrenas laterales (ML) pertenecientes a los complejos morrénicos externos: A) morrena lateral derecha en el sector del frente glaciar, B) bloques cuarcíticos de dimensiones métricas situados en la cresta de la morrena de la fotografía anterior, C) morrenas laterales en las inmediaciones de la Braña de las Cadenas y D) morrena central ligeramente incurvada.

En la margen izquierda del Valle de Refuexu, entre $1.160 \mathrm{~m}$ y $1.380 \mathrm{~m}$ de altitud, se localiza otra morrena lateral de grandes dimensiones $(600 \mathrm{~m}$ de longitud, $50 \mathrm{~m}$ de potencia y 11 ha de superficie ocupada por el till; Figura 2). Su forma es arqueada a favor de la curva que describe dicho valle en su parte final, y se encuentra totalmente recubierta por un hayedo. Esta morrena marca el límite oriental de la lengua afluente que descendió de los dos circos situados al oeste del Pico Ferreirúa y al este del Pico del Águila, y del área del Collado de Ferreirúa, para confluir con la lengua principal, canalizada por el Valle de la Puerca.

En la margen derecha del Valle de la Puerca, concretamente en las inmediaciones de la Braña de Cadenas, se conserva un complejo morrénico integrado por dos cordones laterales (uno interno, y el otro más externo y situado a mayor cota) adosados a la vertiente y con una morfología casi rectilínea. La altitud de la morrena más baja oscila 
entre $1.380 \mathrm{~m}$ y $1.535 \mathrm{~m}$, mientras que la más alta se desarrolla entre $1.280 \mathrm{~m}$ y 1.395 $\mathrm{m}$. Sus espesores son de 26 y $50 \mathrm{~m}$, respectivamente. En conjunto, el complejo morrénico tiene una superficie de 24 ha (Figura 4C). La morrena de mayor altitud está seccionada por un surco de arrollada que depositó parte de sus sedimentos sobre la morrena más baja, dando lugar a un cono de deyección. Sobre la superficie de ambas, que están recubiertas de un matorral muy tupido, se han encontrado bloques dispersos de cuarcita.

La última morrena correspondiente a los complejos morrénicos externos se localiza en la margen izquierda del sector de cabecera del río la Puerca (Figuras 2 y 4D). Tiene una disposición arqueada, desarrollándose entre 1.409 y $1.227 \mathrm{~m}$ de altitud, con una longitud total de $603 \mathrm{~m}$, una potencia de máxima de $36 \mathrm{~m}$ y una extensión de 7 ha. Genéticamente se trata de una morrena central, generada entre dos lenguas confluentes procedentes de los circos generados inmediatamente al Este y al Oeste del Pico del Cuervo.

4.1.2.2. Complejos morrénicos internos. Cabe citar la existencia de cinco complejos morrénicos internos, desconectados de los anteriores y situados a mayores cotas altimétricas, que a su vez están integrados por hasta 7 cordones laterales y tres arcos morrénicos frontales (Figura 2). El primero, integrado por un cordón morrénico, se encuentra adosado a la margen izquierda del Valle de Refuexu. Alcanza $210 \mathrm{~m}$ de longitud, una altitud comprendida entre 1.305 y $1.352 \mathrm{~m}$, y 1,1 ha de extensión. El segundo complejo, que tiene una longitud de $910 \mathrm{~m}$, está situado inmediatamente al este del Pico Ferreirúa. Su altitud oscila entre $1.217 \mathrm{~m}$ y $1.641 \mathrm{~m}$, y cuenta con una superficie de till de 11 ha. Se corresponde con una morrena lateral de desarrollo rectilíneo bastante desdibujada por los procesos postglaciares (el área está afectada por movimientos en masa rápidos, principalmente del tipo de avalanchas de nieve) que afectaron al depósito y a su morfología, ya que apenas conserva aristas morrénicas asociadas. Pese a la abundancia de vegetación arbustiva en el área, se han localizado bloques de cuarcita de dimensiones métricas, y en un corte de una pista forestal que atraviesa la parte distal de la morrena se puede apreciar claramente la estructura típica del till, en el cual se conservan además cantos estriados (Figura 5A).

El siguiente complejo morrénico se localiza a los pies del segundo circo situado al este del Pico Ferreirúa. En total, este complejo está integrado por hasta cinco morrenas laterales bien definidas, dos de ellas depositadas en la margen izquierda y tres en la derecha conforme al flujo del hielo (Figuras 2, 5B y 5C). En cuanto a sus dimensiones, su rango altitudinal está comprendido entre los $1.769 \mathrm{~m}$ y los $1.323 \mathrm{~m}$, con longitudes que abarcan entre 338 y $998 \mathrm{~m}$, potencias que oscilan entre 15 y $40 \mathrm{~m}$, y con una superficie total ocupada por el till de 29 ha. Se trata en todos los casos de cordones morrénicos muy bien definidos y rectilíneos o ligeramente incurvados. Igual que en el caso anterior, este complejo morrénico está recubierto por una vegetación arbustiva muy espesa, pero se han localizado abundantes bloques de cuarcita de tamaño métrico.

Al Norte del Pico del Cuervo se depositó otro complejo morrénico integrado por una morrena muy arqueada aguas abajo, de $412 \mathrm{~m}$ desarrollo longitudinal total y de $20 \mathrm{~m}$ de 
espesor máximo, comprendida en el rango altitudinal de $1.546 \mathrm{~m}$ a $1.441 \mathrm{~m}$ (Figura 5D). En su interior se distingue también un arco morrénico frontal incidido en su parte central, así como abundante till recubriendo toda el área existente entre medias, dando lugar conjuntamente a una formación superficial que interpretamos como perteneciente a un glaciar negro. La superficie total ocupada por el till es de 6 ha. Por último, al NW del pico del Boquerón de Ventana se conserva un pequeño arco morrénico entre $1.504 \mathrm{y}$ $1.545 \mathrm{~m}$ de altitud, con una longitud de $93 \mathrm{~m}$ y 0,4 ha de extensión.
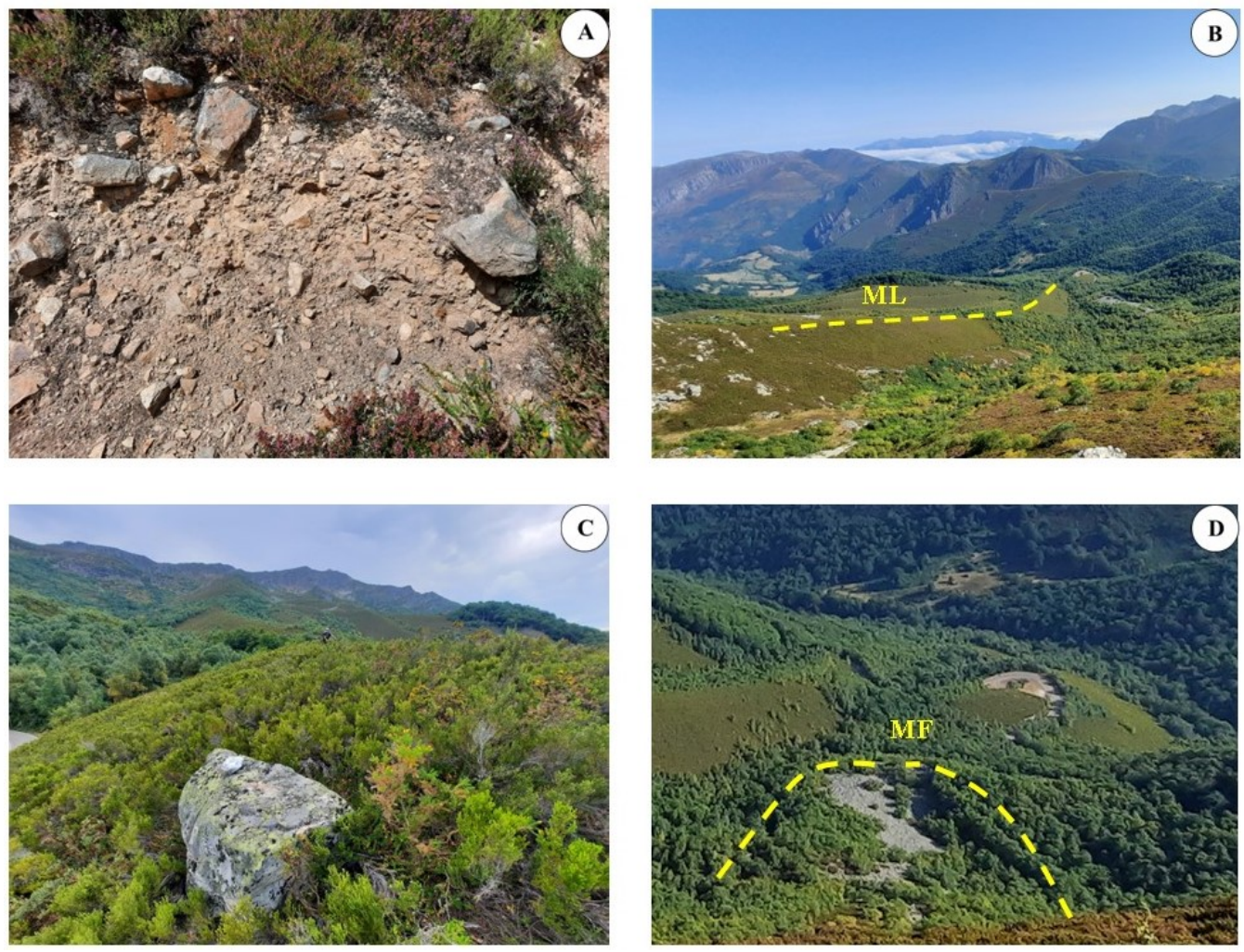

Figura 5. Morrenas laterales (ML) pertenecientes a los complejos morrénicos internos. A) Corte en el que se aprecia la estructura característica del till, situado en el sector distal del complejo morrénico que desciende desde el circo emplazado inmediatamente al Este del Pico Ferreirúa; B) vista de una morrena lateral desde la cima del Pico del Cuervo, C) bloque cuarcítico de dimensiones métricas situado en la cresta de la morrena de la fotografía anterior y D) Arco morrénico frontal (MF) de la lengua que descendía del circo situado al Noroeste del Pico del Cuervo.

4.1.2.3. Complejos morrénicos de pie de circo. La tercera generación de complejos morrénicos está integrada principalmente por cuatro arcos morrénicos simples y uno desdoblado, situados a los pies de los circos glaciares con mejores condiciones topoclimáticas del conjunto estudiado (Figura 2). En el caso del circo labrado en la cara NW del Pico Ferreirúa, se conservan un arco morrénico desdoblado con una longitud total de $238 \mathrm{~m}$ y una superficie de 3 ha, desarrollándose entre 1.582 y $1.674 \mathrm{~m}$ de altitud. En los dos circos contiguos excavados al este del Pico Ferreirúa se conservan dos arcos frontales correspondientes a dos pequeños glaciares de circo. En el caso del situado más al Oeste de ambos, el arco tiene una longitud de $118 \mathrm{~m}$ y una superficie de till de 3,5 ha, discurriendo entre las altitudes de 1.526 y 1.618 m (Figura 6A). Por su 
parte, el más oriental, incidido en su área central, está dividido en dos lomas de 123 y $84 \mathrm{~m}$ de longitud, con una superficie muy similar al anterior (3,6 ha), emplazándose entre 1.544 y $1.614 \mathrm{~m}$. Este último tiene un glaciar rocoso en su interior, que será tratado en posteriores apartados (Figura 6B).

Por último, en los dos circos situados al Este y el Oeste del Pico del Cuervo se han generado otras dos morrenas de menor entidad. La más occidental, de disposición arqueada, está también dividida por los procesos erosivos en dos partes (longitudes de 128 y 64 m), y ocupa únicamente 1,3 ha, discurriendo entre las cotas de 1.560 a 1.586 $\mathrm{m}$. Por su parte, la más oriental constituye un postizo de till de morfología escasamente definida, que alcanza $102 \mathrm{~m}$ de longitud y una superficie de 0,4 ha. Su rango altimétrico va de $1.587 \mathrm{a} 1.650 \mathrm{~m}$.
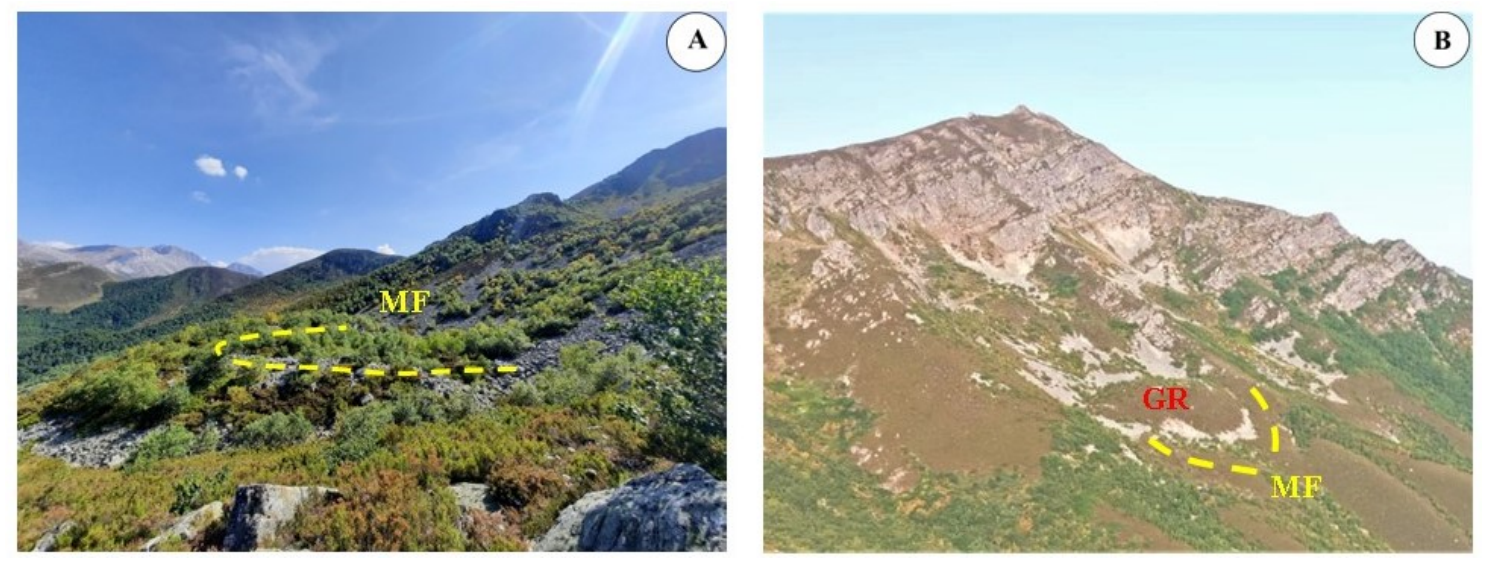

Figura 6. Arcos morrénicos frontales (MF) situados en los dos circos labrados al Este del Pico Ferreirúa, pertenecientes a los complejos morrénicos de pie de circo: A) vista del arco morrénico más occidental, B) y del más oriental, que cuenta con un glaciar rocoso ubicado en una posición más interna (GR).

\subsubsection{Fases glaciares, reconstrucción de los paleoglaciares y cálculo de paleoELAs}

A partir de los complejos morrénicos descritos anteriormente, es posible reconstruir tres fases en la evolución glaciar de la vertiente asturiana del Puerto de Ventana: fase de máxima extensión de los hielos (MEH), fase interna y fase de circos (Tabla 1). Este mismo modelo evolutivo fue utilizado para describir el glaciarismo en zonas cercanas del suroccidente de Asturias y el noroccidente de León, como es el caso del Valle de Somiedo (MENÉNDEZ-DUARTE, 1995; MENÉNDEZ-DUARTE y MARQUÍNEZ, 1996) y el sector del Alto Sil (SANTOS-GONZÁLEZ, 2010). Este modelo evolutivo también ha sido validado en otros sectores del Macizo Asturiano y las Montañas Cantábricas (FROCHOSO y CASTAÑÓN, 1998; GALLINAR et al., 2014; RODRÍGUEZ PÉREZ, 2015; SANTOS-GONZÁLEZ et al., 2022; SERRANO et al., 2022). Solamente en los Picos de Europa, como conjunto montañoso de mayor altitud del reborde montañoso NW de la Península Ibérica, existe otra cuarta fase glaciar muy reciente: la Pequeña Edad del Hielo. Esta fase, de carácter histórico, ha sido reconstruida a partir de las huellas morfológicas conservadas y del testimonio de viajeros y estudiosos decimonónicos. Los testimonios que se conservan describen 
algunos pequeños glaciares al amparo de paredes de orientación Norte en las cumbres más elevadas (GONZÁLEZ-TRUEBA, 2005, 2007; GONZÁLEZ-TRUEBA et al., 2008; SERRANO et al., 2012, 2013; RUIZ-FERNÁNDEZ et al., 2016, 2022; RUIZFERNÁNDEZ y GARCÍA-HERNÁNDEZ, 2018).

4.1.3.1. Fase de Máxima Expansión de los Hielos (MEH). La fase de MEH se puede reconstruir a partir de los restos que conforman el complejo morrénico externo de Ventana. Así, como se puede apreciar en la reconstrucción glaciar realizada a partir de un modelo digital (Figuras 7A y 8A), la mayor parte del área de estudio estuvo cubierta por los hielos durante la MEH. Se generó un solo aparato glaciar de tipo alpino compuesto, al estar formado por la confluencia de dos lenguas de hielo. La lengua principal descendió desde la cabecera del río la Puerca, estando definida su área de acumulación por la línea de cinco circos glaciares que van desde el Pico del Boquerón de Ventana hasta el sector Norte del Pico Ferreirúa. En esta lengua el espesor máximo del hielo fue de $198 \mathrm{~m}$ (Tabla 1; Figura 8B). Por su parte, la otra lengua descendió por el Valle de Refuexu, siendo su área de acumulación la comprendida entre el Pico Ferreirúa por el Este, el Collado Ferreirúa por el Sur (que actuó como un área de transfluencia glaciar entre la parte asturiana y la leonesa) y el cordal del Pico del Águila por el Noroeste. El espesor máximo alcanzado por esta segunda lengua fue de $178 \mathrm{~m}$. El frente del glaciar se situó a $1.016 \mathrm{~m}$ de altitud, con una superficie total de 491,3 ha y un volumen de hielo de $510,7 \mathrm{hm}^{3}$ (Tabla 1).

La altitud de la paleoELA durante esta fase, según el método AABR, fue de $1.520 \mathrm{~m}$. Con el método AAR la altitud de la paleoELA fue de $1.557 \mathrm{~m}$, mientras que con el THAR fue de $1.400 \mathrm{~m}$. Por tanto, su altitud mediante AABR y AAR es más baja que la obtenida para la MEH en los Picos de Europa, que en este caso fue de $1.600 \mathrm{~m}$, si bien con importantes diferencias entre los tres macizos que integran dicho conjunto montañoso, así como entre unos paleoglaciares y otros en función de factores como su tipología, su orientación y su topografía (GONZÁLEZ-TRUEBA, 2007; SERRANO et al., 2012, 2013).

Si bien se está trabajando en la obtención de un marco cronológico preciso de la evolución glaciar del sector estudiado mediante dataciones de isótopos cosmogénicos, por el momento se carece de resultados. No obstante, por correlación con otros sectores de las Montañas Cantábricas en los que sí existen edades absolutas, la MEH precedería cronológicamente al Último Máximo Glaciar Global (UMGG), oscilando entre 45 y 36 ka (MIS 3; ej. JIMÉNEZ y FARIAS, 2002; MORENO et al., 2010; SERRANO et al., 2012, 2013; JIMÉNEZ et al., 2013; PELLITERO, 2013; RUIZ-FERNÁNDEZ et al., 2016, 2022; RUIZ-FERNÁNDEZ y GARCÍA-HERNÁNDEZ, 2018). No obstante, cada vez son más frecuentes en las Montañas Cantábricas los estudios que evidencian fases glaciares previas, con edades que están en la horquilla de 65 a 78 ka (MIS 4; FROCHOSO et al., 2013), en torno a 110 ka (MIS 5d) en el Valle del Porma, e incluso de $\sim 170-150$ ka para los vestigios más antiguos, probablemente heredados de una glaciación anterior durante el MIS 6 (RODRÍGUEZ-RODRÍGUEZ et al., 2016, 2018). 
A partir de la aplicación de dataciones cosmogénicas de $\mathrm{Ne}^{21}$, VIDAL-ROMANÍ et al. $(1999,2015)$ también señalan la existencia de fases glaciares antiguas en dos sierras de las Montañas del Noroeste (Queixa-Invernadoiro y Gerês-Xurés), en un rango de 238 a $126 \mathrm{ka}$.

Tabla 1. Parámetros morfométricos y paleoELAs de los glaciares identificados en el área de estudio en cada fase.

\begin{tabular}{|c|c|c|c|c|c|c|c|c|c|c|c|c|c|}
\hline \multirow[b]{2}{*}{$\begin{array}{l}\text { Fase } \\
\text { Glaciar }\end{array}$} & \multirow[b]{2}{*}{$\begin{array}{c}\text { Nombre } \\
\text { del } \\
\text { glaciar }\end{array}$} & \multirow{2}{*}{$\begin{array}{c}\text { Altitud } \\
\text { máxima } \\
\text { del } \\
\text { circo } \\
(\mathrm{m} \\
\text { s.n.m. })\end{array}$} & \multirow{2}{*}{$\begin{array}{l}\text { Altitud } \\
\text { del } \\
\text { fondo } \\
\text { del } \\
\text { circo } \\
\text { (m } \\
\text { s.n.m.) } \\
\end{array}$} & \multirow{2}{*}{$\begin{array}{l}\text { Altitud } \\
\text { del } \\
\text { frente } \\
\text { glaciar } \\
\text { (m } \\
\text { s.n.m.) }\end{array}$} & \multirow[b]{2}{*}{$\begin{array}{c}\text { Superficie } \\
\text { glaciada } \\
\text { (ha) }\end{array}$} & \multirow[b]{2}{*}{$\begin{array}{c}\text { Volumen } \\
\text { glaciar } \\
\left(\mathrm{hm}^{3}\right)\end{array}$} & \multirow{2}{*}{$\begin{array}{c}\text { Espesor } \\
\text { máximo } \\
\text { del } \\
\text { hielo } \\
(\mathrm{m})\end{array}$} & \multirow{2}{*}{$\begin{array}{l}\text { Longitud } \\
\text { máxima } \\
(\mathrm{km})\end{array}$} & \multirow[b]{2}{*}{$\begin{array}{c}\text { Anchura } \\
\text { Máxima } \\
(\mathrm{Km})\end{array}$} & \multirow[b]{2}{*}{$\begin{array}{c}\text { Dirección } \\
\text { del } \\
\text { glaciar }\end{array}$} & \multicolumn{3}{|c|}{$\begin{array}{c}\text { ELA } \\
\text { (m s.n.m) }\end{array}$} \\
\hline & & & & & & & & & & & $\begin{array}{c}\text { AABR } \\
1,7\end{array}$ & \begin{tabular}{|l|} 
AAR \\
0,65
\end{tabular} & $\begin{array}{c}\text { THAR } \\
0,4\end{array}$ \\
\hline $\begin{array}{c}\text { MEH } \\
\text { (Máxima } \\
\text { extension } \\
\text { del hielo) } \\
\end{array}$ & Ventana & 1.977 & 1.531 & 1.016 & 491,26 & 510,7 & 198 & 3.11 & 1.8 & NW & 1520 & 1557 & 1400 \\
\hline \multirow{5}{*}{$\begin{array}{l}\text { Fase } \\
\text { Interna }\end{array}$} & $\begin{array}{c}\text { Ferreirúa } \\
\text { NW }\end{array}$ & 1.977 & 1.650 & 1.275 & 32 & 8,1 & 51 & 1,34 & 0,48 & NW/NE & 1.599 & 1.563 & $1.555,8$ \\
\hline & $\begin{array}{c}\text { Ferreirúa } \\
\text { NE (I) }\end{array}$ & 1.977 & 1.690 & 1.226 & 35,5 & 10 & 47 & 1,28 & 0,52 & NE & 1.596 & 1.620 & $1.526,4$ \\
\hline & $\begin{array}{c}\text { Ferreirúa } \\
\text { NE (II) }\end{array}$ & 1.977 & 1.645 & 1.272 & 33,5 & 9,8 & 50 & 1,34 & 0,34 & NE & 1.605 & 1.613 & $1.536,6$ \\
\hline & $\begin{array}{c}\text { Cuervo } \\
\text { NW }\end{array}$ & 1.757 & 1.531 & 1.427 & 33 & 14 & 50 & 0,82 & 0,48 & $\mathrm{~N}$ & 1.604 & 1.649 & 1.559 \\
\hline & $\begin{array}{c}\text { Cuervo } \\
\text { NE }\end{array}$ & 1.757 & 1.542 & 1.355 & 35 & 7,9 & 42 & 1,18 & 0,46 & $\mathrm{~N}$ & 1.573 & 1.584 & $1.515,8$ \\
\hline \multirow{5}{*}{$\begin{array}{l}\text { Fase de } \\
\text { Circos }\end{array}$} & $\begin{array}{c}\text { Ferreirúa } \\
\text { NW }\end{array}$ & 1.977 & 1.800 & 1.638 & 11 & 4,1 & 33 & 0,42 & 0,32 & NW & 1.731 & 1.744 & $1.773,6$ \\
\hline & $\begin{array}{c}\text { Ferreirúa } \\
\text { NE (I) }\end{array}$ & 1.977 & 1.800 & 1.746 & 17 & 4,3 & 36 & 0,53 & 0,40 & NE & 1.702 & 1.712 & $1.838,4$ \\
\hline & $\begin{array}{c}\text { Ferreirúa } \\
\text { NE (II) }\end{array}$ & 1.977 & 1.800 & 1.569 & 16 & 5,2 & 34 & 0,59 & 0,24 & NE & 1.696 & 1.723 & $1.732,2$ \\
\hline & $\begin{array}{c}\text { Cuervo } \\
\text { NW }\end{array}$ & 1.757 & 1.800 & 1.594 & 7 & 3,1 & 27 & 0,41 & 0,23 & $\mathrm{~N}$ & 1.692 & 1.703 & $1.659,2$ \\
\hline & $\begin{array}{l}\text { Cuervo } \\
\text { NE }\end{array}$ & 1.757 & 1.800 & 1.615 & 5,6 & 2,3 & 21 & 0,31 & 0,16 & $\mathrm{~N}$ & 1.650 & 1.680 & $1.671,8$ \\
\hline
\end{tabular}

En otros sectores montañosos de la Península Ibérica, la MEH de la última Glaciación antecede también al UMGG, como por ejemplo en las Montañas del Noroeste y en Sierra Nevada, donde se han obtenido cronologías anteriores a $30 \mathrm{ka}$ cal BP en el primer caso, y de 30-32 ka en el segundo (PÉREZ-ALBERTI et al., 2011; GÓMEZ-ORTIZ et al., 2012, 2015). En los Pirineos, el uso de dataciones de C14 y OSL en depósitos de origen glaciar, o relacionados mofoestratigráficamente con depósitos y formas glaciares, permiten inferir igualmente una MEH previa al UMMG (ej. GARCÍA-RUIZ et al., 2003, 2013; GONZÁLEZ-SAMPÉRIZ et al., 2006; JIMÉNEZ et al., 2013). Por su parte, la reciente generalización de las dataciones mediante isótopos cosmogénicos también ha ofrecido resultados más próximos o incluso sincrónicos con el UMGG para la MEH en los Pirineos de entre 26 y 23 ka (ej. PALLÀS et al., 2006; DELMAS et al., 2008; GARCÍA-RUIZ et al., 2014; PALACIOS et al., 2015), y también en el Sistema Central (ej. PALACIOS et al., 2012a, 2012b). 


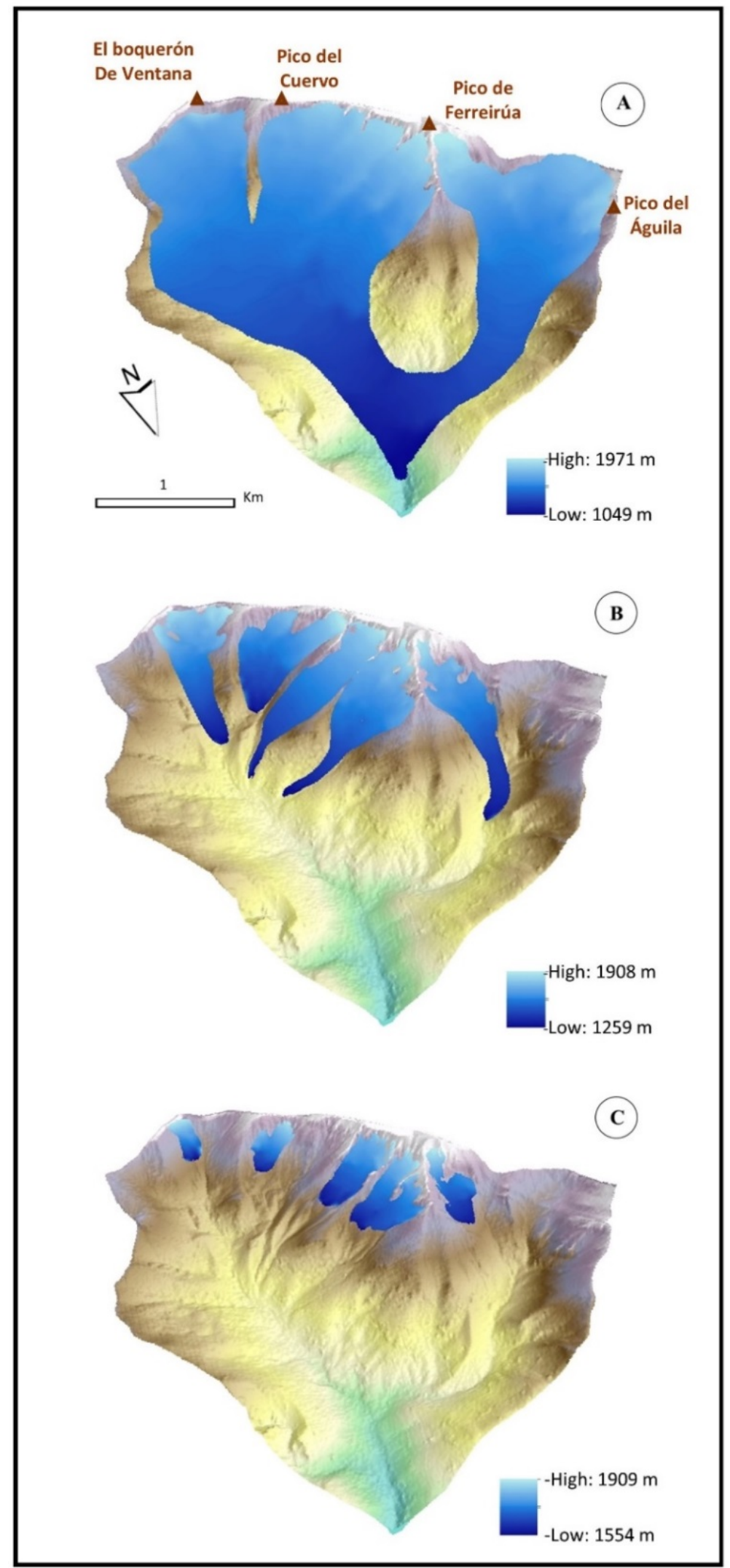

Figura 7. Vista en 3D de las tres fases de avance glaciar identificadas del Puerto de Ventana dentro de la Última Glaciación: A) fase de MEH; B) fase interna; y C) fase de circos. 
Por ello, existe una descoordinación temporal entre diversos conjuntos montañosos peninsulares que podría estar motivada por diferencias de escala regional dentro de la dinámica atmosférica desarrollada durante la Última Glaciación, a la que responderían rápidamente los pequeños glaciares de montaña debido a su alta sensibilidad climática (FLORINETH y SCHLÜTER, 2000; KUHLEMANN et al., 2008). Así, según DOMÍNGUEZ-VILLAR et al. (2013), los glaciares de montaña del centro peninsular alcanzan su extensión máxima en torno a $26 \mathrm{ka}$ como respuesta a un aumento de las precipitaciones entre 29 y $25 \mathrm{ka}$, junto a condiciones de insolación mínimas. Por su parte, en los Pirineos DELMAS et al. (2015) señala la existencia de patrones de reavance diferentes durante el MIS 2 entre las áreas de influencia climática atlántica y mediterránea de esta cordillera. En cualquier caso, no debemos olvidar la incertidumbre derivada del uso de diferentes sistemas de datación (GARCÍA-RUIZ et al., 2010; JIMÉNEZ et al., 2013).

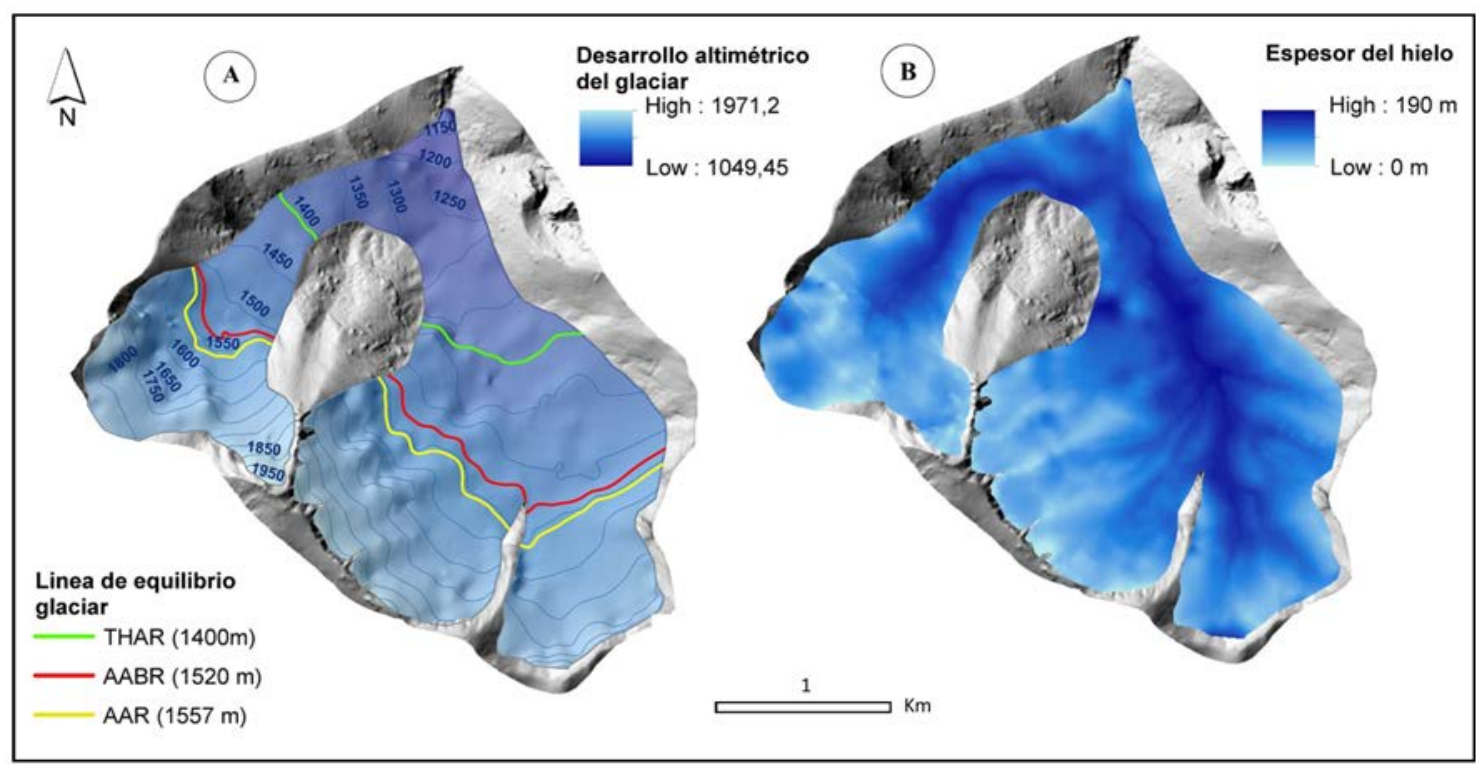

Figura 8. Reconstrucción del paleoglaciar de Ventana durante la fase de MEH. A) Desarrollo altimétrico del glaciar con indicación de las paleoELAs calculadas mediante los métodos AABR, AAR y THAR. B) Espesor del hielo.

4.1.3.2. Fase interna. La fase interna ha sido reconstruida a partir de los límites marcados por los complejos morrénicos internos. Se trata de una nueva fase de avance y estabilización glaciar precedida de un retroceso significativo del primitivo paleoglaciar de Ventana, hasta el punto de quedar subdividido en cinco glaciares alpinos menores (Figura 7B). El primero descendió del circo situado al oeste del Pico Ferreirúa. Tenía una longitud de $1,34 \mathrm{~km}$, con una superficie total de 32 ha y un espesor máximo del hielo de $51 \mathrm{~m}$ (Tabla 1). La segunda de las lenguas, generada a partir del primer circo situado al este del Pico Ferreirúa y de otro situado justo al Norte del anterior, tenía una longitud de 1,28 km, y contó con una superficie de 35,5 ha y un espesor de $47 \mathrm{~m}$. La tercera se desarrolló en el segundo circo situado al este del Pico Ferreirúa, y contó con una longitud de $1,34 \mathrm{~km}$, una superficie de 33,5 hectáreas y un espesor de $50 \mathrm{~m}$. Por su 
parte, la cuarta lengua, que descendía del circo situado al noroeste del Pico del Cuervo, no tuvo un gran desarrollo longitudinal si la comparamos con los anteriores $(0,82 \mathrm{~km})$, con una superficie de 33 ha y un espesor máximo del hielo de $50 \mathrm{~m}$ (Tabla 1). La quinta y última lengua, se corresponde con el paleoglaciar que descendió del circo situado al noreste del Pico del Cuervo. En este caso la lengua glaciar alcanzó 1,18 km de largo, una superficie de 35 ha y un espesor máximo del hielo de $42 \mathrm{~m}$. El volumen total del hielo durante esta fase fue de 49,8 $\mathrm{hm}^{3}$, lo que supone una pérdida del 90,25\% del volumen del hielo con respecto a la fase de MEH. El valor de las paleoELAs durante la Fase Interna según el método AABR osciló entre 1.573 m y 1.605 m (Tabla 1), mientras que con el método AAR lo hizo entre 1.563 y $1.649 \mathrm{~m}$, y con el THAR entre $1.515 \mathrm{y}$ $1.559 \mathrm{~m}$ (Tabla 1). Los datos aportados se corresponden con el momento de máxima extensión de los cinco glaciares dentro de la Fase Interna. No obstante, a partir de la sucesión de cordones y arcos pertenecientes a los complejos morrénicos internos, se pueden reconstruir hasta tres pulsaciones menores dentro de ella.

Como en la fase de $\mathrm{MEH}$, se carece por el momento de dataciones absolutas del área del Puerto de Ventana para esta segunda etapa. Sin embargo, en las Montañas Cantábricas, donde la Fase Interna está ampliamente representada, su cronología ha sido inferida a partir de secuencias sedimentarias lacustres y fluviotorrenciales, e incluso mediante dataciones de isótopos cosmogénicos (JIMÉNEZ y FARIAS, 2002; JIMÉNEZ et al., 2013; PELLITERO, 2013; SERRANO et al., 2012, 2013; RODRÍGUEZ-RODRÍGUEZ et al., 2015, 2016, 2017, 2018). Las edades obtenidas en los citados estudios se encuentran dentro de la horquilla de 23 a $17 \mathrm{ka}$, de ahí que dicho avance sea coetáneo al UMGG (MIS 2). Esta fase está presente también en los principales macizos ibéricos, como Sierra Nevada, donde se ha detectado un reavance glaciar entre 19 y $20 \mathrm{ka}$ (GÓMEZ-ORTIZ et al., 2012, 2015). También ha sido identificada en los Pirineos (PALLÀS et al., 2006; DELMAS et al., 2008, 2011; PALACIOS et al., 2015), o el Sistema Central (PALACIOS et al., 2012a, 2012b; CARRASCO et al., 2013; DOMÍNGUEZ-VILLAR et al., 2013), en un rango temporal de 23 a 17 ka; así como en las Montañas del Noroeste (VIDAL-ROMANí et al., 1999, 2015), en este caso con edades de 21-23 ka.

4.1.3.3. Fase de Circos. Por último, la Fase de Circos se ha podido reconstruir a partir de los complejos morrénicos de pie de circo. En efecto, durante la tercera y última fase de avance glaciar, en la vertiente asturiana del Puerto de Ventana existieron también cinco pequeños glaciares de circo (Figura 7C). El primero de ellos, situado a los pies del circo localizado al noroeste del Pico Ferreirúa, tuvo una longitud de 0,42 km, una superficie de 11 ha y un espesor máximo del hielo de $33 \mathrm{~m}$. El segundo glaciar, emplazado en el primer circo al este del Pico Ferreirúa, contó con una longitud de 0,53 $\mathrm{km}$, una superficie de 17 ha y un espesor máximo del hielo de $36 \mathrm{~m}$ (Tabla 1). El tercero, localizado inmediatamente al Este del anterior, presentó una longitud de 0,59 $\mathrm{km}$, una superficie de 16 ha y un espesor máximo de $34 \mathrm{~m}$. El cuarto glaciar, generado al noroeste del Pico del Cuervo, contó con una longitud de 0,41 km, una superficie cubierta por el hielo de 7 ha, así como un espesor máximo de $27 \mathrm{~m}$. Por último, el 
glaciar ubicado en el circo situado al noreste del Pico del Cuervo es el que presenta las dimensiones más reducidas, con $0,31 \mathrm{~km}$ de desarrollo longitudinal, una superficie de 5,6 ha, y un espesor máximo del hielo de $21 \mathrm{~m}$ (Tabla 1; Figuras 2 y 7C). El volumen total del hielo para la Fase de Circos fue de $19 \mathrm{hm}^{3}$, lo que supuso una reducción del 96,28 \% del volumen total del hielo con respecto a la fase de MEH. En este caso, las paleoELAs oscilaron según el método AABR entre $1.650 \mathrm{~m}$ y $1.731 \mathrm{~m}$, y mediante los métodos AAR y THAR entre 1.680 y 1.744, así como entre 1.659 y 1.838 m, respectivamente (Tabla 1 ).

La fase de glaciares de circo también ha sido ampliamente identificada en diferentes conjuntos altimontanos de las Montañas Cantábricas, como por ejemplo en los Picos de Europa, Fuentes Carrionas, el Macizo de las Ubiñas, la Sierra de Cebolleda, Alto Campo y Valdecebollas, así como el Alto Nalón y el Alto Porma (JIMÉNEZ, 1996; GONZÁLEZ-TRUEBA, 2007; PELLITERO, 2013; SERRANO et al., 2012, 2013; GALLINAR et al., 2014; RODRÍGUEZ-RODRÍGUEZ et al., 2016, 2017, 2018; RUIZFERNÁNDEZ et al., 2016, 2022; RUIZ-FERNÁNDEZ y GARCÍA-HERNÁNDEZ, 2018). Los datos inferidos a partir del estudio de registros lacustres en el lago Enol (Macizo Occidental de los Picos de Europa) por MORENO et al. (2010), y en Campomayor (entre los macizos Central y Oriental de los Picos de Europa) por SERRANO et al. (2012), permiten identificar una fase fría entre 13,9 y 11,6 ka cal BP, de ahí que tanto estos trabajos como otros posteriores (ej. RUIZ-FERNÁNDEZ et al., 2016, 2022; RUIZ-FERNÁNDEZ y GARCÍA-HERNÁNDEZ, 2018), atribuyan la fase de avance glaciar existente a los pies de los grupos de cumbres más elevadas de los tres macizos de los Picos de Europa a dicha horquilla temporal (especialmente al Younger Dryas, periodo frío acontecido entre 12,9 y 11,7 ka; RASMUSSEN et al., 2014).

Sin embargo, en otros conjuntos montañosos del Macizo Asturiano de menor vigor altimétrico, como el Alto Porma, las dataciones obtenidas a partir de isótopos cosmogénicos evidencian que las últimas fases de recesión glaciar tuvieron lugar aquí en torno a 17,7 ka (RODRÍGUEZ-RODRÍGUEZ et al., 2016). Posteriormente, el área quedó bajo el influjo de condiciones periglaciares, como evidencia el glaciar rocoso datado en las cercanías del Lago Ausente, cuya estabilización (en su área distal) aconteció hace 15,7 ka (RODRÍGUEZ-RODRÍGUEZ et al., 2016). Por su parte, en el Valle de Monasterio (cuenca del Alto Nalón) las fases glaciares más recientes han sido datadas en 14 ka (RODRÍGUEZ-RODRÍGUEZ et al., 2017). Según estos autores, a partir de entonces se produjo una transición gradual hacia condiciones periglaciares, existiendo glaciares rocosos que mantuvieron su actividad hasta $13 \mathrm{ka}$. Por tanto, a la espera de la obtención de edades absolutas a partir de dataciones cosmogénicas de $\mathrm{Be}^{10}$, la adscripción cronológica provisional de esta fase en el Puerto de Ventana presenta $a$ priori mayor incertidumbre, pudiendo abarcar una amplia horquilla temporal dentro del periodo comprendido entre el final del UMGG y el Holoceno (Termination-1; DENTON et al., 2010), que incluye fases frías como el Oldest Dryas (17,5-14,6 ka) y el ya mencionado Younger Dryas, y otras más cálidas como el interestadial BøllingAllerød, desarrollado entre 14,6 y 12,9 ka; RASMUSSEN et al., 2014). 


\subsection{La morfodinámica periglaciar y nival}

En primer lugar, cabe citar formas del relieve como un glaciar rocoso y tres campos de bloques (Figura 2; Tabla 2). En ambos casos, se trata de geoformas cuya génesis está directamente relacionada con la presencia de permafrost y, por tanto, son morfologías relictas, heredadas de periodos fríos finipleistocenos. El glaciar rocoso se localiza inmediatamente al Este del Pico Ferreirúa. Es de tipo lobado o de lengua, cuenta con una orientación NE y está integrado por hasta cuatro arcos principales. Su anchura máxima es de $211 \mathrm{~m}$, su longitud de $346 \mathrm{~m}$ y su superficie de 5,24 ha. Por su parte, los campos de bloques se sitúan también en las proximidades del Pico Ferreirúa, concretamente en el área culminante del cordal, si bien ya en su caída hacia la vertiente leonesa. Conjuntamente, los tres campos de bloques, integrados por bloques masivos de cuarcita de dimensiones decimétricas a métricas, tienen extensiones que oscilan entre 0,16 y 0,71 ha, con una superficie total de 1,74 ha (Figura 9A; Tabla 2).
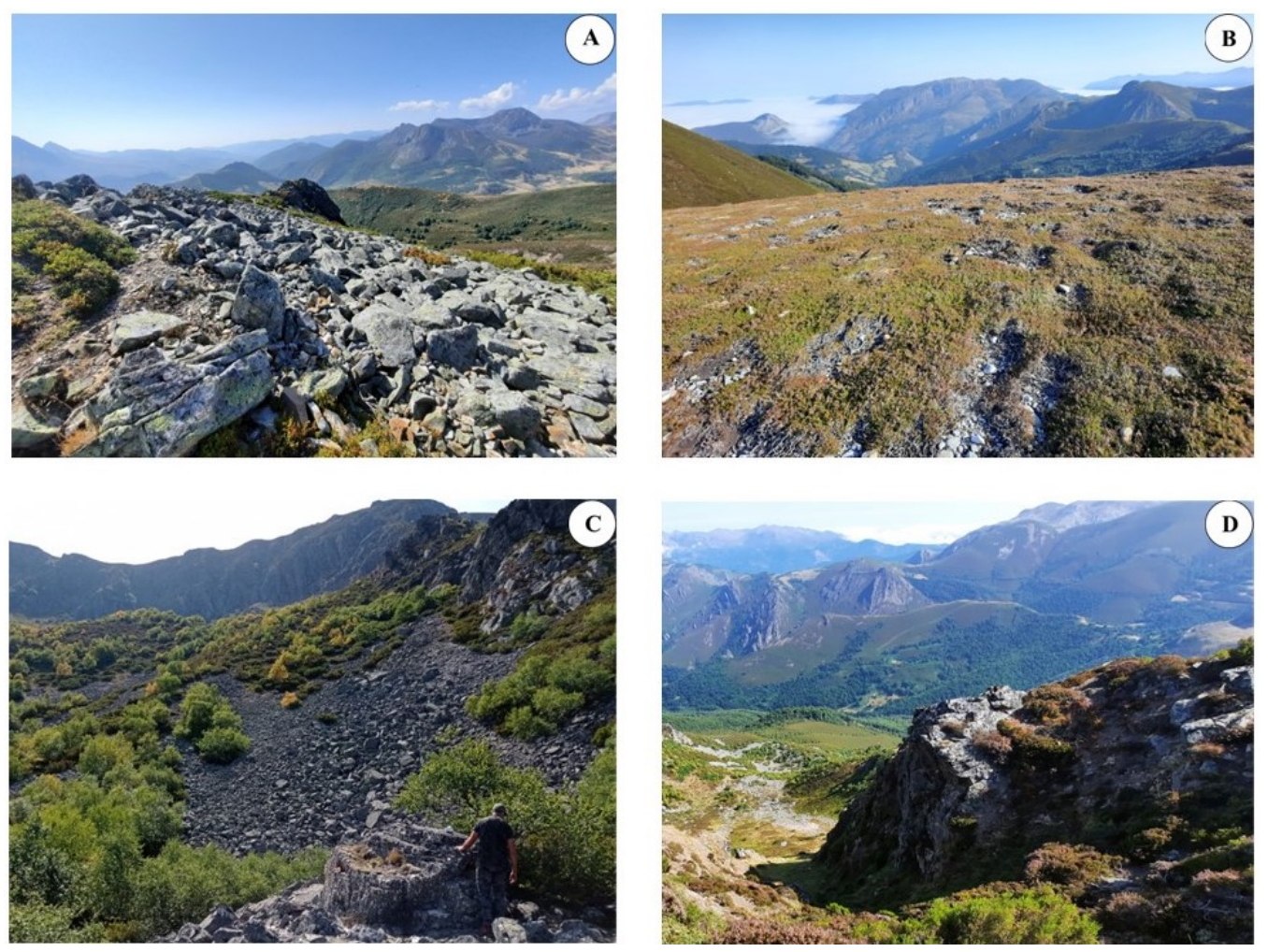

Figura 9. Formas periglaciares y nivales en el área de estudio: A) Detalle de un campo de bloques relicto en las cercanías del Pico Ferreirúa, B) suelos ordenados en las inmediaciones del Pico del Cuervo, C) talud de derrubios en el que se diferencian zonas activas de otras inactivas (cara noreste del Pico Ferreirúa), y D) canal de aludes con forma de tobogán en la vertiente norte del Pico Ferreirúa.

En un rellano del cordal culminante existente entre las cumbres del Pico del Cuervo y el Boquerón de Ventana, se ha observado la presencia de suelos ordenados (Figuras 2 y 9B). Éstos se caracterizan por su disposición en forma de círculos elongados y de bandas longitudinales o figuras lineales, en los que se da una alternancia de sectores 
integrados por matriz arenosa y otros por materiales gruesos (gravas y cantos). Recubriendo a los primeros se desarrolla una formación rastrera de brecina (Calluna vulgaris).

Tabla 2. Formas periglaciares y nivales identificadas en el área de estudio.

\begin{tabular}{|c|c|c|}
\hline $\begin{array}{c}\text { Formas y } \\
\text { depósitos } \\
\text { periqlaciares }\end{array}$ & Localización & Características \\
\hline Glaciar rocoso & $\begin{array}{l}\text { Situado en el interior del } \\
\text { circo que se encuentra al } \\
\text { NE del Pico Ferreirúa. }\end{array}$ & $\begin{array}{l}\text { Morfología relicta integrada por arcos y surcos en los que } \\
\text { afloran bloques de cuarcita de tamaños decimétricos a } \\
\text { métricos. Su altitud oscila entre los } 1.622 \mathrm{~m} \text { del frente y los } \\
1.714 \mathrm{~m} \text { de la raíz, que enlaza con un talud de derrubios. Tiene } \\
5,25 \text { ha de extensión, } 346 \text { m de longitud y } 211 \text { de anchura. Se } \\
\text { encuentra parcialmente recubierto de vegetación de porte } \\
\text { arbustivo y arborescente. }\end{array}$ \\
\hline $\begin{array}{l}\text { Campos de } \\
\text { bloques }\end{array}$ & $\begin{array}{l}\text { Ubicados en las } \\
\text { inmediaciones S y SE del } \\
\text { Pico Ferreirúa, es decir, } \\
\text { en su vertiente leonesa. }\end{array}$ & $\begin{array}{l}\text { Depósitos de bloques de tamaño métrico a decimétrico, de } \\
\text { carácter relicto. Presentan extensiones que oscilan entre } 0,16 \text { y } \\
0,71 \text { ha, y se sitúan a una altitud media de } 1.932 \mathrm{~m} \text {. La } \\
\text { superficie total que ocupan es de } 1,74 \text { ha. }\end{array}$ \\
\hline Canales de aludes & $\begin{array}{l}\text { Situados en la paredes de } \\
\text { los circos labradas desde } \\
\text { el NW del Pico del } \\
\text { Cuervo hasta el NW del } \\
\text { Pico Ferreirúa. }\end{array}$ & $\begin{array}{l}\text { Presentan una morfología en tobogán y están labradas a favor } \\
\text { de las fracturas presentes en las cuarcitas de la Fm. Barrios } \\
\text { (Ordovícico). Se han contabilizado un total de } 23 \text { canales. Las } \\
\text { pendientes oscilan entre } 39^{\circ} \text { y } 42^{\circ} \text {. Se trata de canales cortas, } \\
\text { con una longitud media de } 64 \mathrm{~m} \text {. El punto más alto de las } \\
\text { canales oscila entre } 1.924 \text { y } 1.674 \mathrm{~m} \text {, y el más bajo entre } 1.832 \\
\text { y } 1.653 \mathrm{~m} \text {. }\end{array}$ \\
\hline $\begin{array}{l}\text { Taludes de } \\
\text { derrubios }\end{array}$ & $\begin{array}{l}\text { Situados en las laderas } \\
\text { de la línea de cumbres } \\
\text { existente entre el Pico del } \\
\text { Cuervo y el Pico del } \\
\text { Águila (preferentemente } \\
\text { en las vertientes de los } \\
\text { respectivos circos } \\
\text { glaciares). }\end{array}$ & $\begin{array}{l}\text { Formas integradas por gravas, cantos y bloques con escasa } \\
\text { matriz fina en superficie (principalmente aflora en sus bordes). } \\
\text { Sus derrubios están ordenados generalmente de acuerdo con } \\
\text { una secuencia granocreciente entre las áreas proximales y las } \\
\text { distales. Tienen distinto grado de actividad. En las partes } \\
\text { medias y distales predominan los sectores inactivos, } \\
\text { recubiertos de vegetación. Estos sectores alcanzan una } \\
\text { extensión total de } 29,85 \text { ha y una pendiente media de } 24,6^{\circ} \text {. } \\
\text { Los sectores activos (sin recubrimiento vegetal) están } \\
\text { asociados a procesos como la caída de aludes, la formación de } \\
\text { flujos de derrubios, la acción de la arroyada y la solifluxión, } \\
\text { contando por tanto con presencia de otras morfologías } \\
\text { (principalmente relacionadas con la solifluxión). Su extensión } \\
\text { es de } 10,6 \text { ha, y su pendiente media de } 36,2^{\circ} \text {. }\end{array}$ \\
\hline Suelos ordenados & $\begin{array}{l}\text { Localizados en un collado } \\
\text { existente entre las } \\
\text { cumbres del Boquerón de } \\
\text { Ventana y el Pico del } \\
\text { Cuervo. }\end{array}$ & $\begin{array}{l}\text { Se trata de círculos elongados incipientes y bandas } \\
\text { longitudinales de tamaño decimétrico a métrico, generados en } \\
\text { ámbitos de topografía horizontal o suevamente inclinada, a } \\
\text { favor de sectores de matriz fina que alternan con otros } \\
\text { integrados por materiales gruesos (gravas y cantos). Los finos } \\
\text { están recubiertos por brezal. Ocupan una extensión de } \sim 0,5 \text { ha } \\
\text { (longitud de } 245 \mathrm{~m} \text { y anchura de } 111 \mathrm{~m} \text { ). Se sitúan a unos } \\
1.703 \mathrm{~m} \text { de altitud. }\end{array}$ \\
\hline $\begin{array}{l}\text { Lóbulos de } \\
\text { solifluxión }\end{array}$ & $\begin{array}{l}\text { Localizados en los bordes } \\
\text { de los canchales que } \\
\text { recubren el interior de los } \\
\text { circos. }\end{array}$ & $\begin{array}{l}\text { Depósitos de morfología lobular y dimensiones variables } \\
\text { (decimétricas a métricas), generadas a favor de materiales } \\
\text { finos. }\end{array}$ \\
\hline $\begin{array}{c}\text { Césped } \\
\text { almohadillado }\end{array}$ & $\begin{array}{l}\text { Localizados en los bordes } \\
\text { de los canchales que } \\
\text { recubren el interior de los } \\
\text { circos. }\end{array}$ & $\begin{array}{l}\text { Formas de tamaño decimétrico arquedas en planta y } \\
\text { recubiertas de vegetación herbácea, que aparecen } \\
\text { escalonadas en los sectores con mayor abundancia de matriz } \\
\text { fina de los taludes y conos de derrubios. }\end{array}$ \\
\hline
\end{tabular}


Por otro lado, también están presentes los conos y taludes de derrubios (Figuras 2, 9C y 10; Tabla 2), desarrollados en el interior de los circos y alimentados por canales de aludes generalmente cortas y rectilíneas, al estar generadas a favor de fracturas existentes en las cuarcitas ordovícicas (Figuras 2 y 9D; Tabla 2). Éstas últimas, que cuentan con un perfil típico en tobogán, se concentran en la vertiente asturiana de la cabecera glaciar que articula los picos Ferreirúa y Cuervo, en la que se han identificado un total de 23 canales, en su mayoría recubiertos por vegetación herbácea. Se trata de canales de fuerte pendiente (entre $39^{\circ}$ y $42^{\circ}$ ), que durante el invierno y el inicio de la primavera generan avalanchas de nieve que afectan a las partes proximales de los conos y taludes de derrubios.

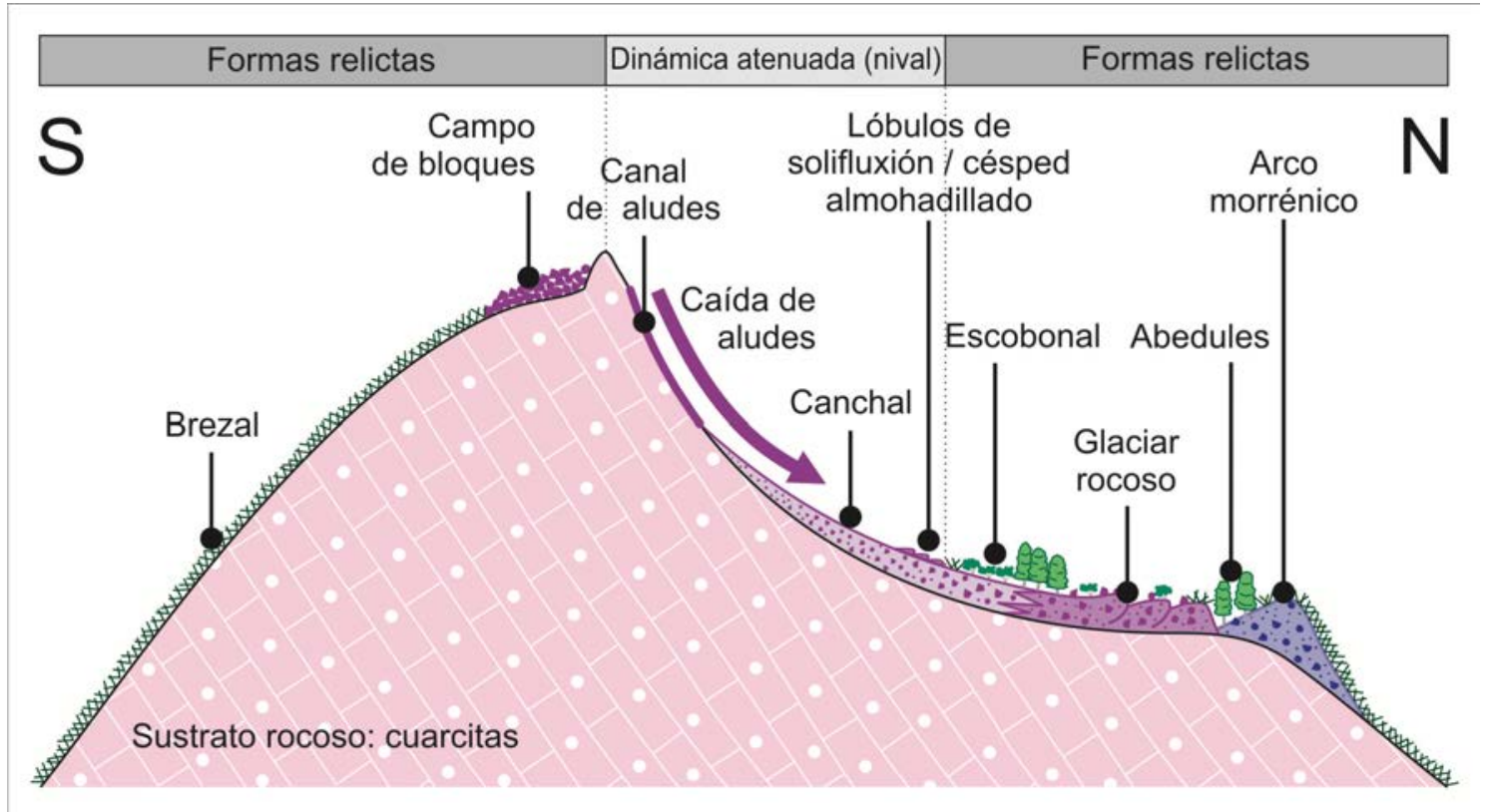

Figura 10. Perfil geomorfológico idealizado de la dinámica periglaciar relicta y actual propia del Puerto de Ventana.

Estos últimos están ordenados siguiendo secuencias granocrecientes entre las partes proximales y distales. En general, actualmente presentan escasa actividad. Buena parte de la superficie total de estas formas (concretamente 29,85 ha, lo que supone el 73,8 \% del total cartografiado), está colonizada por vegetación, representada principalmente por especies como arándanos (Vaccinium myrtillus), diversos brezos, enebros (Juniperus communis) y abedules (Betula celtiberica). En otras ocasiones, los derrubios que las integran presentan una coloración más oscura que las áreas activas, por encontrarse masivamente recubiertos de líquenes (principalmente Rhizocarpon geograficum), evidenciando que su dinámica es nula o muy reducida. Se trata, principalmente, de las áreas distales y de los bordes de los conos y taludes de derrubios. La pendiente media de dichos sectores es de 24,6 ${ }^{\circ}$. En cambio, las partes activas tienen una coloración más blanquecina, fruto de la fractura reciente de los clastos y de su movimiento vertiente abajo. En este caso, la inclinación media es mayor $\left(36,2^{\circ}\right)$, al situarse preferentemente en los sectores proximales y medios de los conos y taludes. Sin embargo, su superficie 
total es menor (10,57 ha, lo que supone el $26,2 \%$ de la extensión total de estas geoformas).

En el interior de algunos taludes de derrubios, sobre todo en aquellos donde hay una mayor abundancia de fracción fina, se han generado pequeños lóbulos de solifluxión (Tabla 2), céspedes almohadillados, y bloques de tamaño decimétrico que fluyen ladera abajo a favor de capas de materiales más finos, generando un abultamiento de finos en su frente, conforme al sentido del movimiento. No obstante, no cuentan con el surco posterior típico de los bloques aradores.

En definitiva, parte de las formas y depósitos periglaciares estudiados en este trabajo son relictas, heredadas de condiciones paleoclimáticas más frías que las actuales, en las que se llegó a formar permafrost, necesario para la génesis de glaciares rocosos y campos de bloques (Figura 10). A su vez, la presencia de estas morfologías está condicionada por la litología existente. Es sabido, por ejemplo, que los glaciares rocosos son geoformas con un alto control litológico (REDONDO-VEGA et al., 2004; JOHNSON et al., 2007; SANTOS-GONZÁLEZ, 2010; GÓMEZ-VILLAR et al., 2011; PELLITERO et al., 2011), por lo que son muy abundantes sobre las cuarcitas paleozoicas del Macizo Asturiano. Por el contrario, son muy escasos sobre las calizas del Carbonífero, motivo por el cual hay muy pocos glaciares rocosos relictos en ámbitos como por ejemplo los Picos de Europa (GONZÁLEZ-TRUEBA, 2007; PELLITERO et al., 2011; RUIZ-FERNÁNDEZ, 2015).

El resto de las morfologías y depósitos muestran una actividad geomorfológica atenuada, vinculada principalmente a procesos nivales (caída de aludes, fusión del manto nival y consiguiente saturación hídrica de las formaciones superficiales), como acontece en el caso de los taludes y conos de derrubios, y de otras formas asociadas a ellos (Figura 10). En menor medida, este ambiente también está afectado por ciclos de hielo-deshielo de corta duración (esencialmente diaria), lo que tiene implicaciones en la movilización de partículas dentro de las formaciones superficiales y a lo largo de las vertientes; así como en la actuación de la gelifracción, si bien su actividad es reducida en comparación con otras áreas de las Montañas Cantábricas, debido al moderado desarrollo altimétrico del Puerto de Ventana. En los canchales también se ha detectado la actuación de procesos como la arroyada difusa y la formación de pequeños flujos de derrubios. Estos últimos se dan en relación con la saturación hídrica de las formaciones superficiales, debido a la fusión nival primaveral, o bien a partir de episodios tormentosos intensos (GONZÁLEZ-TRUEBA, 2007; RUIZ-FERNÁNDEZ et al., 2014). Es necesario tener en cuenta, además, que la actividad de las formas periglaciares funcionales es estacional, y que frecuentemente se halla favorecida por la actuación de otros procesos que no son propiamente periglaciares.

Por tanto, los sectores culminantes del Puerto de Ventana se encuadran dentro del piso nivoperiglaciar, desarrollado en el Macizo Asturiano entre 1.800 y $2.200 \mathrm{~m}$ y ampliamente representado en muchos otros sectores montañosos del mismo, como el Puerto de San Isidro (RODRÍGUEZ PÉREZ, 1995) y el Macizo del Sinclinal de Saliencia (RODRÍGUEZ PÉREZ, 2009). Sin embargo, el área de estudio no alcanza la 
altitud suficiente como para que en ella se den procesos periglaciares más intensos, propios del piso crionival, caracterizado por un mayor dinamismo en comparación con el anterior. En las Montañas Cantábricas, el piso crionival se desarrolla por encima $2.200 \mathrm{~m}$ en conjuntos montañosos como los Picos de Europa, Fuentes Carrionas o las Ubiñas (BROSCHE, 1978; CASTAÑÓN y FROCHOSO, 1998; SERRANO y GONZÁLEZ-TRUEBA, 2004A; GONZÁLEZ-TRUEBA, 2007; PELLITERO, 2012; GALLINAR et al., 2014; RUIZ-FERNÁNDEZ et al., 2014, 2016; 2017).

\section{CONCLUSIONES}

Esta investigación presenta los resultados del estudio de la evolución glaciar y la morfodinámica periglaciar del Puerto de Ventana. Se han identificado tanto las formas de origen glaciar de tipo erosivo (circos, umbrales, rocas aborregadas, estrías, y una cubeta de sobreexcavación glaciar), como depósitos glaciares en forma de cordones y arcos (organizados en tres generaciones de complejos morrénicos distintos: externos, internos y de pie de circo). A partir de la identificación de estas geoformas, así como del establecimiento de sus relaciones morfoestratigráficas, se han reconstruido tres fases en la evolución glaciar de la vertiente asturiana del Puerto de Ventana: Fase de MEH, Fase Interna y Fase de Circos.

En la primera fase (MEH) el Puerto de Ventana estuvo ocupado por un solo aparato glaciar, con una superficie total de 491,3 ha y un volumen de hielo de $510,7 \mathrm{hm}^{3}$. En esta fase la mayor parte del área de estudio estuvo cubierta por los hielos y la altitud de la paleoELA se situó, en función del método de cálculo, entre 1.400 y $1.557 \mathrm{~m}$. Las dataciones obtenidas en zonas cercanas de las Montañas Cantábricas coinciden en que la MEH precede cronológicamente al UMGG, y oscila entre 45 y 36 ka. Posteriormente, el paleoglaciar previamente desarrollado en la vertiente asturiana del Puerto de Ventana experimentó un fuerte retroceso. Por consiguiente, en la Fase Interna existieron cinco glaciares alpinos menores, reduciéndose en un 90,25 \% el volumen del hielo que se alcanzó con respecto a la fase anterior. El valor de las paleoELAs osciló en este caso entre 1.515 y $1.649 \mathrm{~m}$. La mayor parte de los trabajos con geocronologías realizados en las Montañas Cantábricas coinciden en que esta fase es contemporánea al UMGG, con edades en el rango 23 a 17 ka. Finalmente, durante la Fase de Circo, el área de estudio contó con cinco pequeños glaciares de circo, oscilando las paleoELAs entre $1.650 \mathrm{~m} \mathrm{y}$ $1.838 \mathrm{~m}$. En esta última etapa, el volumen del hielo se había reducido un 96,28\% respecto a la fase de $\mathrm{MEH}$. Hasta la fecha, los datos cronológicos atribuibles tanto a esta fase como a las anteriores son inexistentes en el Puerto de Ventana, constituyendo este punto una de las líneas claves a desarrollar en próximos trabajos de investigación.

En cuanto a la morfodinámica periglaciar y nival, se han identificado morfologías relictas, relacionadas con la antigua presencia de permafrost (un glaciar rocoso y tres campos de bloques), cuya génesis estuvo altamente condicionada por la litología existente en el área (cuarcitas paleozoicas en el área de cumbres). Entre las morfologías 
funcionales, cuyas dinámicas tendrán un carácter estacional, aparecen suelos ordenados incipientes, conos y taludes de derrubios y geoformas de origen solifluidal. En todo caso, hay que destacar lo atenuado de su actividad geomorfológica actual y la discreta acción de la gelifracción, notablemente reducida en comparación con otras áreas de las Montañas Cantábricas. Los procesos nivales serían, por tanto, los que mayor influencia tienen en la evolución actual de estas formas.

\section{AGRADECIMIENTOS}

Benjamín González Díaz agradece el apoyo al programa FPU del Ministerio de Educación, Cultura y Deporte de España para el desarrollo de sus investigaciones (referencia: FPU19/06583). Esta contribución estudia los temas de investigación tratados en el proyecto de investigación de excelencia PID2020-115269GB-I00 (MICINN, Gobierno de España).

\section{BIBLIOGRAFÍA}

Allen, T.C. 1998. Topographic context of glaciers and perennial snowfields, Glacier National Park, Montana. Geomorphology, 21, 207-216. https://doi.org/10.1016/S0169555X(97)00059-7

Alonso, V. 1989. Glaciares rocosos fósiles en el área Degaña-Leitariegos (Occidente de Asturias, Cordillera Cantábrica). Cuaternario y Geomorfología, 3 (1- 4), 9-15.

Alonso, V. 1991. Geomorfología de las cabeceras de los ríos Narcea, Navia y Sil, y del Parque Nacional de la Montaña de Covadonga (NO de la Península Ibérica). Universidad de Oviedo. 366 pp.

Alonso, V. 1994. Análisis de circos glaciares en las cabeceras de los ríos Narcea, Ibias y Sil. Cordillera Cantábrica (NW de la Península Ibérica). Cuaternario y Geomorfología, 8 (1-2), 109-121.

Alonso, V., Flor, G. 1987. Evolución cuaternaria del Valle de Degaña, SO de Asturias. En: Baena, R., Fernández, J.J., Guerrero, I., Eds.: Actas de la VII Reunión sobre el Cuaternario Ibérico, pp. 159-161. AEQUA, Sevilla.

Alonso, V., Trombotto, D. 2009. Periglacial geomorphology of El Miro area, Cantabrian Mountains, NW Spain. Zeitschrift für Geomorphologie, 53 (3), 335-357. https://doi.org/10.1127/0372-8854/2009/0053-0335

Benn, D.I., Owen, L.A., Osmaston, H.A., Seltzer, G.O., Porter, S.C., Mark, B. 2005. Reconstruction of equilibrium-line altitudes for tropical and sub-tropical glaciers. Quaternary International, 138-139, 8-21. https://doi.org/10.1016/j.quaint.2005.02.003

Benn, D.I., Evans, D.J.A. 1998. Glaciers and glaciation. Arnold, Londres, 734 pp.

Benn, D.I., Gemmell, A.M.D. 1997. Calculating equilibrium line altitudes of former glaciers by the balance ratio method: a new computer spreadsheet. Disponible en: https://risweb.standrews.ac.uk/portal/en/researchoutput/calculating-equilibriumline-altitudes-of-formerglaciers-a-new-computer-spreadsheet(426a7587-290c-4a34-b1992b5f96d186f6)/export.html 
Benn, D.I., Lehmkuhl, F. 2000. Mass balance and equilibrium-line altitudes of glaciers in highmountain environments. Quaternary International, 65-66, 15-29. https://doi.org/10.1016/S1040-6182(99)00034-8

Benn, D.I., Owen, L.A., Osmaston, H.A., Seltzer, G.O., Porter, S.C., Mark, B. 2005. Reconstruction of equilibrium-line altitudes for tropical and sub-tropical glaciers. Quaternary International, 138-139, 8-21. https://doi.org/10.1016/j.quaint.2005.02.003

Brosche, K.U. 1978. Formas actuales y límites inferiores periglaciares en la Península Ibérica. Estudios Geográficos, 151, 131-161.

Carrasco, R.M., Pedraza, J., Domínguez-Villar, D., Villa, J., Willenbring, J.K. 2013. The plateau glacier in the Sierra de Béjar (Iberian Central System) during its maximum extent. Reconstruction and chronology. Geomorphology, 196, 83-93. https://doi.org/10.1016/j.geomorph.2012.03.019

Carrivick, J.L., Brewer, T.R. 2004. Improving local estimations and regional trends of glacier $\begin{array}{llll}\text { equilibrium line altitudes. Geografiska Annaler, 86A, } & \text { 67-79. }\end{array}$ https://doi.org/10.1111/j.0435-3676.2004.00214.x

Castañón, J.C. 1983. El glaciarismo cuaternario del Macizo de Ubiña (Asturias-León) y su importancia morfológica. Ería. Revista Cuatrimestral de Geografía, 4, 3-49.

Castañón, J.C., Frochoso, M. 1994. El periglaciarismo de la Cordillera Cantábrica. En: Gómez, A., Simón, M., y Salvador, F., Eds., Periglaciarismo en la Península Ibérica, Canarias y Baleares, Monografías de la S.E.G., nº 7, pp., 75-91. Universidad de Granada, Granada.

Castañón, J.C. 1989. Las formas de relieve de origen glaciar en los sectores central y oriental del Macizo Asturiano. Universidad de Oviedo, Oviedo, 788 p. (microfichas).

Castañón, J.C., Frochoso, M. 1998. La alta Montaña Cantábrica: condiciones térmicas y morfodinámicas en los Picos de Europa. En: Gómez-Ortiz, A., Salvador, F., Schulte, L., García, A., Eds., Procesos biofisicos actuales en medios fríos, pp. 113-132. Universidad de Barcelona, Barcelona.

Cowton, T., Hughes, P.D., Gibbard, P.L. 2009. Paleoglaciation of Parque Natural Lago de Sanabria, northwest Spain. Geomorphology, 108, 282-291. https://doi.org/10.1016/j.geomorph.2009.02.007

Delmas, M. D. 2015. The last maximum ice extent and subsequent deglaciation of the Pyrenees: an overview of recent research. Cuadernos de Investigación Geográfica/Geographical Research Letters, 41, 359-387. https://doi.org/10.18172/cig.2708

Delmas, M., Gunnell, Y., Braucher, R., Calvet, M., Bourlès, D. 2008. Exposure age chronology of the last glaciation in the Eastern Pyrenees. Quaternary Research, 69, 231-241. https://doi.org/10.1016/j.yqres.2007.11.004

Denton, G.H., Anderson, R.F., Toggweiler, J.R., Edwards, R.L., Schaefer, J.M., Putnam, A.E. 2010. The last glacial termination. Science, 328 (5986), 1652-1656. https://doi.org/10.1126/science.1184119

Domínguez-Villar, D., Carrasco, R.M., Pedraza, J., Cheng, H., Edwards, R.L., Willenbring, J.K. 2013. Early maximum extent of paleoglaciers from Mediterranean mountains during the last glaciation. Scientific Reports, 3, 20-34. https://doi.org/10.1038/srep02034

Felicísimo, A.M., Alonso, V. 1988. Formas erosivas glaciares y radiación solar: un ensayo de correlación en el valle de Ibias (Degaña, Asturias). Trabajos de Geología, 17, 19-31.

Florineth, D., Schlüchter, C. 2000. Alpine evidence for atmospheric circulation patterns in Europe during the Last Glacial Maximum. Quaternary Research, 54, 295-308. https://doi.org/10.1006/qres.2000.2169

Frochoso, M. 1980. El Macizo Central de los Picos de Europa y sus glaciares. Ería, 1, 67-87. 
Frochoso, M. 1990. Geomorfología del Valle del Nansa. Universidad de Cantabria, Santander, $281 \mathrm{p}$.

Frochoso, M., Castañón, J.C. 1998. El relieve glaciar de la Cordillera Cantábrica. En: GómezOrtiz, A., Pérez-Alberti, A., Eds.: Las huellas glaciares de las montañas españolas, pp. 65137. Universidad de Santiago de Compostela, Santiago de Compostela.

Frochoso, M., González, R., Allende, F. 2013. Pleistocene glacial morphology and timing of Last Glacial Cycle in Cantabrian Mountains (Northern Spain): new chronological data from the Asón Area. Central European Journal of Geoscience, 5, 12-27. https://doi.org/10.2478/s13533-012-0117-8

Furbish, D.J., Andrews, J.T. 1984. The use of hypsometry to indicate long-term stability and response of valley glaciers to changes in mass transfer. Journal of Glaciology, 30, 199-211. https://doi.org/10.3189/S0022143000005931

Gale, S. J., Hoare, P. G. 1997. The glacial history of the northwest Picos de Europa of northern Spain. Zeitschrift für Geomorphologie N. F., 41, 81-96. https://doi.org/10.1127/zfg/41/1997/81

Gallinar, D., García-Hernández, C., Ruiz-Fernández, J. 2019. Conocimiento histórico y patrimonialización de un enclave de la Montaña Cantábrica: las Ubiñas. Papeles de Geografia, 65, 7-29. https://doi.org/10.6018/geografia.372061

Gallinar, D., Ruiz-Fernández, J., Poblete, M.A., Fernández, A., García, C., Beato, S., Marino, J.L. 2014. Morfología y evolución glaciar en el sector asturiano del Macizo de las Ubiñas. En: Schnabel, S., Gómez, A., Eds.: Avances de la Geomorfología en España 2012-2014, pp. 543-546. Sociedad Española de Geomorfología, Cáceres.

García de Celis, A. 1991. Los glaciares rocosos de la Sierra del Suspirón (León). Polígonos, Revista de Geografia, 1, 9-20.

García de Celis, A. 1997. El relieve de la Montaña Occidental de León. Valladolid, Secretariado de Publicaciones de la Universidad de Valladolid, 290 p

García de Celis, A., Martínez Fernández, L. 2002. Morfología glaciar de las montañas de la cuenca alta de los ríos Sil, Omaña, Luna y Bernesga: revisión y nuevos datos (Montaña Occidental de León). En: Redondo, J., González-Gutierrez, R.B., Carrera, P., Eds., El modelado de origen laciar en las montañas leonesas, pp. 137-196. Universidad de León, León.

García-Ruiz, J.M., Martí, C., Peña, J.L., Sancho, C. Rhodes, E., Valero, B., González, P., Moreno, A. 2012. Glacial and fluvial deposits in the Aragón Valley, Central-Western Pyrenees: chronology of the Pyrenean Late Pleistocene glaciers. Geografiska Annaler, 95A, 15-32. https://doi.org/10.1111/j.1468-0459.2012.00478.x

García-Ruiz, J.M., Moreno, A., González, P., Valero, B., Martí, C. 2010. La cronología del último ciclo glaciar en las montañas del Sur de Europa. Una revisión. Cuaternario y Geomorfología, 24 (1-2), 35-46.

García-Ruiz, J.M., Palacios, D., de Andrés, N., Valero, B.L., López, J.I., Sanjuán, Y. 2014. Holocene and 'Little ice age' glacial activity in the Marboré Cirque, Monte Perdido Massif, Central Spanish Pyrenees. The Holocene, 24 (11), 1439-1452. http://dx.doi.org/10.1177/0959683614544053

García-Ruiz, J.M., Valero, B., Martí, C., González, P. 2003. Asynchroneity of maximum glacier advances in the central Spanish Pyrenees. Journal of Quaternary Science, 18 (1), 61-72. https://doi.org/10.1002/jqs.715

Gómez-Ortiz, A., Oliva, M., Palacios, D., Salvador-Franch, F., Vázquez-Selem, L., SalvàCatarineu, M., de Andrés, N. 2015. The deglaciation of Sierra Nevada (Spain), synthesis of 
the knowledge and new contributions. Cuadernos de Investigación Geográfica, 41(2), 409426. https://doi.org/10.18172/cig.2722

Gómez-Ortiz, A., Palacios, D., Palade, B., Vázquez-Selem, L., Salvador-Franch, F. 2012. The deglaciation of the Sierra Nevada (southern Spain). Geomorphology, 159-160, 93-105. https://doi.org/10.1016/j.geomorph.2012.03.008

Gómez-Villar, A., González-Gutierrez, R.B., Redondo-Vega, J.M., Santos-González, J. 2011. Distribución de glaciares rocosos relictos en la Cordillera Cantábrica. Cuadernos de Investigación Geográfica, 37(2), 49-80. https://doi.org/10.18172/cig.1256

Gómez-Villar, A., Santos-González, J., González-Gutiérrez, R.B., Redondo-Vega, J. M. 2015. Glacial cirques in the southern side of the Cantabrian Mountains of southwestern Europe. Geografiska Annaler: Series A, Physical Geography, 97(4), 633-651. https://doi.org/10.1111/geoa.12104

González-Gutiérrez, R.B. 2002. El relieve de los valles del Torio y Curueño (Montaña Cantábrica Leonesa). Universidad de León, León, 267 p.

González-Sampériz, P., Valero, B.L., Moreno, A., Jalut, G., García-Ruiz, J.M., Martí, C., Delgado, A., Navas, A., Otto, T., Dedoubat, J.J. 2006. Climate variability in the Spanish Pyrenees during the last $30.000 \mathrm{yr}$ revealed by the El Portalet sequence. Quaternary Research, 66, 38-52. https://doi.org/10.1016/j.yqres.2006.02.004

González-Trueba, J.J. 2005. La Pequeña Edad del Hielo en los Picos de Europa (Cordillera Cantábrica, NO de España). Análisis morfológico y reconstrucción del avance glaciar histórico. Cuaternario y Geomorfología, 19 (3-4), 79-94.

González-Trueba, J.J. 2007. Geomorfología del Macizo Central del Parque Nacional Picos de Europa. OAPN, Ministerio de Medio Ambiente, Madrid, $231 \mathrm{pp}$.

González-Trueba, J.J., Martín, R., Martínez, E., Serrano, E. 2008. Little Ice Age glaciation and current glaciers in the Iberian Peninsula. The Holocene, 18 (4), 551-568. https://doi.org/10.1177/0959683608089209

González-Trueba, J.J., Serrano, E. 2010. La nieve en los Picos de Europa. Cuadernos de Investigación Geográfica, 36 (2), 61-84. https://doi.org/10.18172/cig.1238

Jiménez, M. 1996. El glaciarismo en la cuenca alta del río Nalón: una propuesta de evolución de los sistemas glaciares cuaternarios en la Cordillera Cantábrica. Revista de la Sociedad Geológica de España, 9, 157-168.

Jiménez, M., Farias, P. 2002. New radiometric and geomorphologic evidences of a Last Glacial Maximum older than $18 \mathrm{ka}$ in SW European mountains: the example of Redes Natural Park (Cantabrian Mountains, NW Spain). Geodinamica Acta, 15, 93-101. https://doi.org/10.1080/09853111.2002.10510741

Jiménez, M., Rodríguez-Rodríguez, L., García-Ruiz, J.M., Domínguez, M.J., Farias, P., Valero, B., Moreno, A., Rico, M., Valcárcel, M. 2013. A review of glacial geomorphology and chronology in northern Spain: timing and regional variability during the last glacial cycle. Geomorphology, 196, 50-64. https://doi.org/10.1016/j.geomorph.2012.06.009

Johnson, B.G., Thackray, G.D., Van Kirk, R. 2007. The effect of topography, latitude, and lithology on rock glacier distribution in the Lemhi Range, Central Idaho, USA. Geomorphology, 91, 38-50. https://doi.org/10.1016/j.geomorph.2007.01.023

Joly, F. 1997. Glossaire de géomorphologie. Base de données sémiologiques pour la cartographie. Armand Colin, París, 325 p.

Kuhlemann, J., Rohling, E., Krumrei, P., Ivy-Ochs, S., Kucera, M. 2008. Regional sintesis of Mediterranean atmospheric circulation during the Last Glacial Maximum. Science, 321, 1338-1340. https://doi.org/10.1126/science. 1157638 
Meierding, T. C. 1982. Late pleistocene glacial equilibrium-lien altitudes in the Colorado Front Range: a comparison of methods. Quaternary Research, 18, 289-310.

Menéndez Duarte, R. 1995. Geomorfología del área de Somiedo (Cordillera Cantábrica, N de España): aplicaciones de los sistemas de información geográfica al estudio del relieve. Universidad de Oviedo.

Menéndez Duarte, R., Marquínez, J. 1996. Glaciarismo y evolución tardiglaciar de las vertientes en el Valle de Somiedo. Cordillera Cantábrica. Cuaternario y Geomorfología, 10(3-4), 2131 .

Moreno, A., Valero, B.L., Jiménez, M., Domínguez, M.J., Mata, M.P., Navas, A., González, P., Stoll, H., Farias, P., Morellón, M., Corella, J.P., Rico, M. 2010. The last deglaciation in the Picos de Europa National Park (Cantabrian Mountains, Northern Spain). Journal of Quaternary Science, 25 (7), 1076-1091. https://doi.org/10.1002/jqs. 1265

Muñoz Jiménez, J. 1980. Morfología estructural y Glaciarismo Cuaternario en la Cordillera Cantábrica: el relieve del Sinclinal de Saliencia (Asturias-León). Ería. Revista Cuatrimestral de Geografia, 1, 35-66.

Muñoz Jiménez, J. 1982. Geografía Física. El relieve, el clima y las aguas. En: Geografía de Asturias, Tomo I, Quirós F., Ed. Ayalga Ediciones, Madrid, 271p.

Oliva, M., Palacios, D., Fernández-Fernández, J.M. 2022. Iberia, Land of Glaciers. How the mountains were shaped by glaciers. Ed. Elsevier, Amsterdam - Oxford - Cambridge, 597 p.

Oliva, M., Serrano, E., Gómez-Ortiz, A., González-Amuchastegui, M.J., Nieuwendam, A., Palacios, D., Pérez-Alberti, A., Pellitero-Ondicol, R., Ruiz-Fernández, J., Valcárcel M., Vieira, G., Antoniades, D. 2016. Spatial and temporal variability of periglaciation of the Iberian Peninsula. Quaternary Science Reviews, 137, 176-199. https://doi.org/10.1016/j.quascirev.2016.02.017

Osmaston, H. 2005. Estimates of glacier equilibrium line altitudes by the Area $\mathrm{x}$ Altitude, the Area x Altitude Balance Ratio and the Area x Altitude Balance Index methods and their validation. Quaternary International, 22-31. https://doi.org/10.1016/j.quaint.2005.02.004

Palacios, D., Andrés, N., Marcos, J., Vázquez, L. 2012a. Glacial landforms and their paleoclimatic significance in Sierra de Guadarrama, Central Iberian Peninsula. Geomorphology, 139-140, 67-78. https://doi.org/10.1016/j.geomorph.2011.10.003

Palacios, D., Andrés, N., Marcos, J., Vázquez, L. 2012b. Maximum glacial advance and deglaciation of the Pinar Valley (Sierra de Gredos, Central Spain) and its significance in the Mediterranean context. Geomorphology, 177-178, 51-61. https://doi.org/10.1016/j.geomorph.2012.07.013

Palacios, D., Gómez, A., de Andrés, N., Vázquez, L., Salvador, F., Oliva, M. 2015. Maximum extent of late Pleistocene glaciers and last deglaciation of La Cerdanya mountains, Southeastern Pyrenees. Geomorphology, 231, 116-129. https://doi.org/10.1016/j.geomorph.2014.10.037

Pallàs, R., Rodes, A., Braucher, R., Carcaillet, J., Ortuno, M., Bordonau, J., Bourles, D., Vilaplana, J.M., Masana, E., Santanach, P. 2006. Late Pleistocene and Holocene glaciation in the Pyrenees: a critical review and new evidence from 10Be exposure ages, south-central Pyrenees. Quaternary Science Reviews, 25, 2937-2963. https://doi.org/10.1016/j.quascirev.2006.04.004

Pellitero, R. 2009. Application of an alpine geomorphological mapping system to an Atlantic Mountain environment: The Curavacas Massif (Cantabrian Range, Northwest Spain). Journal of Maps, 5, 194-205. https://doi.org/10.4113/jom.2009.1065 
Pellitero, R. 2012. Geomorfología, paleoambiente cuaternario y geodiversidad en el Macizo de Fuentes Carrionas Montaña Palentina. Tesis Doctoral (inédita), Universidad de Valladolid.

Pellitero, R. 2013. Evolución finicuaternaria del glaciarismo en el macizo de Fuentes Carrionas (Cordillera Cantábrica), propuesta cronológica y paleoambiental. Cuaternario y Geomorfología, (1-2), 71-90.

Pellitero, R., Rea, B., Spagnolo, M., Bakke, J., Ivy-Ochs, S., Frew, C.R., Hughes, P., Ribolini, A., Lukas, S., Renssen, H. 2016. GlaRe, a GIS tool for reconstruct the 3D surface of $\begin{array}{llll}\text { palaeoglaciers. Computers and } \quad \text { Geosciences, } & \text { 94, }\end{array}$ https://doi.org/10.1016/j.cageo.2016.06.008

Pellitero, R., Serrano, E., González-Trueba, J.J. 2011. Glaciares rocosos del sector central de la Montaña Cantábrica: indicadores paleoambientales. Cuadernos de Investigación Geográfica, 37 (2), 119-144. https://doi.org/10.18172/cig.1259

Pisabarro, A., Pellitero, R., Serrano, E., Gómez-Lende, M., González-Trueba, J.J. 2017. Ground temperatures, landforms and processes in an Atlantic Mountain. Cantabrian Mountains (Northern Spain). Catena, 149, 623-636. https://doi.org/10.1016/j.catena.2016.07.051

Porter, S.C. 1975. Glaciation limit in the New Zealand's Southern Alps. Artic and Alpine Research, 7, 33-37.

Rasmussen, S.O., Bigler, M., Blockley, S.P., Blunier, T., Buchardt, S.L., Clausen, H.B., Cvijanovic, I., Dahl-Jensen, D., Johnsen, S.J., Fischer, H., Gkinis, V., Guillevic, M., Hoek, W.Z., Lowe, J.J., Pedro, J.B., Popp, T., Seierstad, I.K., Steffensen, J.P., Svensson, A.M., Vallelonga, P., Vinther, B.M., Walker, M.J.C., Wheatley, J.J., Winstrup, M. 2014. A stratigraphic framework for abrupt climatic changes during the Last Glacial period based on three synchronized Greenland ice-core records: refining and extending the INTIMATE event stratigraphy. Quaternary Science Reviews, 106, 14-28. https://doi.org/10.1016/j.quascirev.2014.09.007.

Rea, B.R. 2009. Defining modern day Area-Altitude Balance Ratios (AABRs) and their use in glacier-climate reconstructions. Quaternary Science Reviews, 28, 237-248. https://doi.org/10.1016/j.quascirev.2008.10.011

Redondo-Vega, J.M., Gómez-Villar, A., González-Gutiérrez, R.B. 2004. Localización y caracterización morfométrica de los glaciares rocosos relictos de la Sierra de Gistredo (Montaña Cantábrica, León). Cuadernos de Investigación Geográfica, 30, 35-60. https://doi.org/10.18172/cig.1134

Rodríguez Pérez, C. 1995. Estudio geomorfológico del Puerto de San Isidro. Ería, 36, 63-87.

Rodríguez Pérez, C. 1998. Las formas de relieve y la evolución geomorfológica de la sierra de Sobia (área central de Asturias). Ería,46, 131-147.

Rodríguez Pérez, C. 2012. La evolución geomorfológica de la media montaña cantábrica: las sierras cuarcíticas de Porcabezas y de Santa Cristina (área central de Asturias). Ería, 84-85, 47-75.

Rodríguez Pérez, C. 2015. El relieve de la Montaña Central Asturiana: La Sierra de Sobia y el Macizo de Somiedo. Real Instituto de Estudios Asturianos, 154 pp.

Rodríguez-Rodríguez, L. Jiménez, M., Domínguez, M.J., Rinterknecht, V., Pallàs, R., Bourlès, D. 2016. Chronology of glaciations in the Cantabrian Mountains (NW Iberia) during the Last Glacial Cycle based on in situ-produced 10Be. Quaternary Science Reviews, 138, 3148. https://doi.org/10.1016/j.quascirev.2016.02.027

Rodríguez-Rodríguez, L., Domínguez-Cuesta, M.J., Rinterknecht, V., Jiménez-Sánchez, M., González-Lemos, S., Léanni, L., Sanjurjo, J., Ballesteros, D., Valenzuela, P., Llana-Fúnez, S., ASTER Team. 2018. Constraining the age of superimposed glacial records in mountain environments with multiple dating methods (Cantabrian Mountains, Iberian Peninsula). 
Quaternary Science Reviews, $\quad 195, \quad 215-231$. https://doi.org/10.1016/j.quascirev.2018.07.025

Rodríguez-Rodríguez, L., Jiménez-Sánchez, M., Domínguez-Cuesta, M.J., Aranburu, A. 2015. Research history on glacial geomorphology and geochronology of the Cantabrian Mountains, north Iberia $\left(43-42^{\circ} \mathrm{N} / 7-2^{\circ} \mathrm{W}\right)$. Quaternary International, 364, 6-21. https://doi.org/10.1016/j.quaint.2014.06.007.

Rodríguez-Rodríguez, L., Jiménez-Sánchez, M., Domínguez-Cuesta, M. J., Rinterknecht, V., Pallàs, R., ASTER TEAM. 2017. Timing of last deglaciation in the Cantabrian Mountains (Iberian Peninsula; North Atlantic Region) based on in situ-produced 10Be exposure dating. Quaternary Science Reviews, 171, 166-181. https://doi.org/10.1016/j.quascirev.2017.07.012

Ruiz-Fernández, J. 2011. Geomorphological map of an Atlantic mid-height mountain area: the Juan Robre and Jana Ridge (Cantabrian Range, Northwest Spain). Journal of Maps, 7 (1), 260-272. https://doi.org/10.4113/jom.2011.1173

Ruiz-Fernández, J. 2015. Las formas de modelado glaciar, periglaciar y fluviotorrencial del Macizo Occidental de los Picos de Europa (Cordillera Cantábrica). Boletín de la Asociación de Geógrafos Españoles, 68, 581-587.

Ruiz-Fernández, J., García-Hernández, C. 2018. Morfología y evolución glaciar en el Macizo del Cornión (Picos de Europa, Montañas Cantábricas). Cadernos del Laboratorio Xeolóxico de Laxe, 40, 29-68. https://doi.org/10.17979/cadlaxe.2018.40.0.4912

Ruiz-Fernández, J., González-Díaz, B., Gallinar Cañedo, D., García-Hernández, C. (2022) The glaciers of the Central-Western Asturian Mountains. En: Oliva, M., Palacios, D., Fernández-Fernández, J.M., Eds.: Iberia, Land of Glaciers. How the mountains were shaped by glaciers, pp. 265-288. Elsevier, Amsterdam - Oxford - Cambridge. https://doi.org/10.1016/B978-0-12-821941-6.00013-X.

Ruiz-Fernández, J., Oliva, M., Cruces, A., Lopes, V., Freitas, M.C., Andrade, C., GarcíaHernández, C., López-Sáez, J.A., Geraldes, M. 2016. Environmental evolution in the Picos de Europa (Cantabrian Mountains, SW Europe) since the Last Glaciation. Quaternary Science Reviews, 138, 87-104. https://doi.org/10.1016/j.quascirev.2016.03.002

Ruiz-Fernández, J., Oliva, M., Hrbáček, F., Vieira, G., García-Hernández, C. 2017. Soil temperatures in an Atlantic high mountain environment: The Forcadona buried ice patch (Picos de Europa, NW Spain). Catena, 149 (2), 637-647. https://doi.org/10.1016/j.catena.2016.06.037

Ruiz-Fernández, J., Poblete, M.A., García, C. 2014. Características morfoclimáticas y procesos y formas periglaciares actuales en el Macizo Occidental de los Picos de Europa (Cordillera Cantábrica). En: Gómez-Ortiz A., Salvador F., Oliva M., Salvà, M., Eds., Avances, métodos y técnicas en el estudio del periglaciarismo, pp. 91-103. Universidad de Barcelona, Barcelona.

Ruiz-Fernández, J., Poblete, M.A., Serrano-Muela, P., Martí-Bono, C., García-Ruiz, J.M. 2009. Morphometry of glacial cirques in the Cantabrian range (Northwest Spain). Zeitschrift für Geomorphologie 53, 47-68. https://doi.org/10.1127/0372-8854/2009/0053-0047

Santos-González, J. 2010. Glaciarismo y periglaciarismo en el Alto Sil, Provincia de León (Cordillera Cantábrica). Tesis Doctoral (inédita), Departamento de Geografía y Geología de la Universidad de León, León, 689 p.

Santos-González, J., González-Gutiérrez, R., Gómez-Villar, A., Redondo-Vega, J. 2009. Ground thermal regime in the vicinity of relict rock glaciers (Cantabrian Mountains, NW Spain). Finisterra, 87, 35-44. https://doi.org/10.18055/Finis1375

Santos-González, J., Redondo, J.M., González-Gutiérrez, R.B., Gómez-Villar, A. 2013. Applying the AABR method to reconstruct equilibrium-line altitudes from the last glacial 
maximum in the Cantabrian Mountains (SW Europe). Palaeogeography, Palaeoclimatology, Palaeoecology, $\quad 387, \quad 185-199$. https://doi.org/10.1016/j.palaeo.2013.07.025

Santos-González, J., Redondo-Vega, J.M., García-de Celis, A., González-Gutiérrez, R.B., Gómez-Villar, A. 2022. The glaciers of the Leonese Cantabrian Mountains. En: Oliva, M., Palacios, D., Fernández-Fernández, J.M., Eds.: Iberia, Land of Glaciers. How the mountains were shaped by glaciers, pp. 289-314. Ed. Elsevier, Amsterdam - Oxford Cambridge. https://doi.org/10.1016/B978-0-12-821941-6.00014-1

Serrano, E., Gómez-Lende, M., Pisabarro, A. 2022. The glaciers of the western massifs of Cantabria. En: Oliva, M., Palacios, D., Fernández-Fernández, J.M., Eds.: Iberia, Land of Glaciers. How the mountains were shaped by glaciers, pp. 201-219. Ed. Elsevier, Amsterdam - Oxford - Cambridge. https://doi.org/10.1016/B978-0-12-821941-6.00010-4

Serrano, E., González-Trueba, J.J. 2004a. Morfodinámica periglaciar en el grupo Peña Vieja (Macizo Central de los Picos de Europa - Cantabria). Cuaternario y Geomorfología, 18 (34), 73-88.

Serrano, E., González-Trueba, J.J. 2004b. El método AAR para la determinación de paleoELAs: análisis metodológico y aplicación en el Macizo de Valdecebollas (Cordillera Cantábrica). Cuadernos de Investigación Geográfica, 30, 7-34. https://doi.org/10.18172/cig.1133

Serrano, E., González-Trueba, J.J., González-García, M. 2012. Mountain glaciation and paleoclimate reconstruction in the Picos de Europa (Iberian Peninsula, SW Europe). Quaternary Research, 78, 303-314. https://doi.org/10.1016/j.yqres.2012.05.016

Serrano, E., González-Trueba, J.J., Pellitero, R., González-García, M., Gómez-Lende, M. 2013. Quaternary glacial evolution in the Cantabrian Mountains (Northern Spain). Geomorphology, 196, 65-82. https://doi.org/10.1016/j.geomorph.2012.05.001

Truyols, J., Arbizu, M., García Alcalde, J.L., García López, S., Martinez Chacón, M.L., Méndez Bedia, I., Méndez Fernández, C., Menéndez, J.R., Sánchez de Posada, L.C., Soto, F., Truyols Massoni, M., Villa, E., Marcos, A., Pérez Estaún, A., Pulgar, J. A., Bastida, F., Aller, J., Lorenzo, P., Rodríguez Fernández, L. 1982. Memoria del Mapa Geológico de España. La Plaza Teverga. Hoja 77 (12-6). Instituto Geológico y Minero de España, Madrid, $84 \mathrm{p}$.

Vidal-Romaní, J.R., Fernández-Mosquera, D., Marti, K., 2015. The glaciation of Serra de Queixa-Invernadoiro and Serra do Geres-Xurés, NW Iberia. A critical review and a cosmogenic nuclide (10-Be and 21-Ne) chronology. Cadernos do Laboratorio Xeolóxico de Laxe 38, 25-44. https://doi.org/10.17979/cadlaxe.2015.38.0.3681

Vidal-Romaní, J.R., Fernández-Mosquera, D., Marti, K., De Brum-Ferreira, A., 1999. Nuevos datos para la cronología glaciar pleistocena en el NW de la Península Ibérica. Cadernos do Laboratorio Xeolóxico de Laxe 24, 7-29.

Vieira, G. 2008. Combined numerical and geomorphological reconstruction of the Serra da Estrela plateau icefield, Portugal. Geomorphology, 97, 190-207. https://doi.org/10.1016/j.geomorph.2007.02.042 